\title{
BENCHMARKING NUMERICAL METHODS FOR LATTICE EQUATIONS WITH THE TODA LATTICE
}

\author{
DENIZ BILMAN AND THOMAS TROGDON
}

\begin{abstract}
We compare performances of well-known numerical time-stepping methods that are widely used to compute solutions of the doubly-infinite Fermi-Pasta-Ulam-Tsingou (FPUT) lattice equations. The methods are benchmarked according to (1) their accuracy in capturing the soliton peaks and (2) in capturing highly-oscillatory parts of the solutions of the Toda lattice resulting from a variety of initial data. The numerical inverse scattering transform method is used to compute a reference solution with high accuracy. We find that benchmarking a numerical method on puresoliton initial data can lead one to overestimate the accuracy of the method.
\end{abstract}

\section{INTRODUCTION}

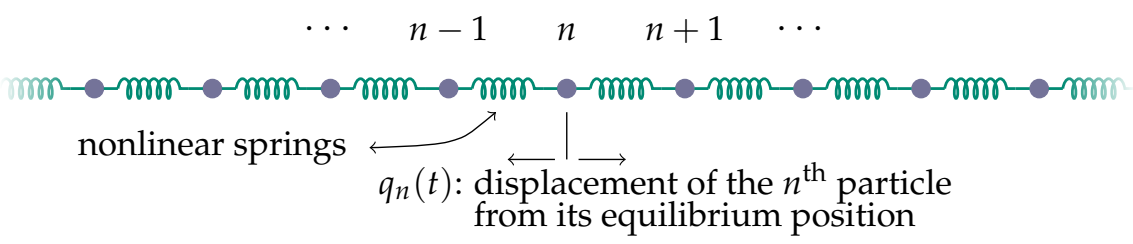

FIGURE 1. One-dimensional chain of particles with nearest neighbor interactions.

Consider the classical problem of one-dimensional infinite chain of particles on a line with nearest-neighbor interactions as depicted in Figure 1. Assume that each particle has unit mass, and that there are no impurities, i.e. the potential energies of the springs between the particles are identical. We let $V: \mathbb{R} \rightarrow \mathbb{R}$ denote the interaction potential between the neighboring particles. With these assumptions, the equations of motion that govern this particle system are given by Newton's Second Law of Motion:

$$
\frac{\mathrm{d}^{2}}{\mathrm{~d} t^{2}} q_{n}=V^{\prime}\left(q_{n+1}-q_{n}\right)-V^{\prime}\left(q_{n}-q_{n-1}\right), \quad n \in \mathbb{Z},
$$

where $q_{n}$ stands for the displacement of the $n^{\text {th }}$ particle from its equilibrium position. Denoting by $p_{n}$ the momentum of the $n^{\text {th }}$ particle, (1) is equivalent to the system of first order differential equations:

$$
\left.\frac{\mathrm{d} p_{n}}{\mathrm{~d} t}=V\left(q_{n+1}-q_{n}\right)\right)-V\left(q_{n}-q_{n-1}\right), \quad \frac{\mathrm{d} q_{n}}{\mathrm{~d} t}=p_{n}, \quad n \in \mathbb{Z}
$$

With the assumptions that $q_{n+1}-q_{n} \rightarrow 0$ and $p_{n} \rightarrow 0$ sufficiently fast as $|n| \rightarrow \infty$ (i.e. no motion at infinity), (2) is a Hamiltonian system of equations

$$
\frac{d p_{n}}{d t}=-\frac{\partial \mathcal{H}(p, q)}{\partial q_{n}}, \quad \frac{d q_{n}}{d t}=\frac{\partial \mathcal{H}(p, q)}{\partial p_{n}}, \quad n \in \mathbb{Z},
$$

Date: September 29, 2017.

2010 Mathematics Subject Classification: 65P10, 65M12, 65M22, 65L06

Keywords: FPUT lattice, Toda lattice, numerical analysis, benchmarking numerical methods, ordinary differential equations, Runge-Kutta methods, symplectic integrators. 
with the Hamiltonian functional $\mathcal{H}(p, q)$ :

$$
\mathcal{H}(p, q)=\sum_{n \in \mathbb{Z}} \frac{1}{2} p_{n}^{2}+V\left(q_{n+1}-q_{n}\right) .
$$

Such nearest-neighbor interacting particle systems with nonlinear interaction forces (anharmonic potentials) include the systems studied in the famous experiment at Los Alamos by Fermi, Pasta, Ulam, and Tsingou [9] in 1953, which a decade later led to discovery of solitons by Zabusky and Kruskal [32]. Such lattice equations model various physical phenomena with a multitude of applications [25]. From a purely mathematical perspective these lattices are used to investigate Poincaré recurrence, chaos, and nonlinear wave phenomena (interaction of solitary waves, solitary wave resolution, see [3] and the references therein). The FPUT-type systems (2) are still an active area of mathematical research $[11,13,31]$.

The particular choice $V(r)=V_{\text {Toda }}(r):=e^{-r}+r-1$ in (2) results in an infinite dimensional, continuous time - discrete space completely integrable system: the celebrated Toda lattice [26]. The main purpose of this work is to compare the performances of various well known numerical time-stepping methods that are widely used to compute the solution of the Cauchy initial value problem for (1). The accuracy of a time-stepping method is most easily inspected when there are exact solutions available at hand, which is of course, rarely the case. Often, numerical analysts make use of nonlinear integrable wave models that possess classes of explicit solutions (e.g. the Toda lattice, the Korteweg-deVries (KdV) equation or the nonlinear Schrödinger (NLS) equation). Then one has the luxury of being able to test their numerical scheme against exact formulae. Many of these infinite-dimensional integrable systems feature dispersive radiation and/or oscillatory tails. This is a critically important ${ }^{1}$ oscillatory component of the solution that decays slowly to the background as $t \rightarrow \infty$. It is often the case that exact solutions formulae are for solitary waves such as breathers or solitons - coherent structures that are localized in space without oscillatory tails or dispersive radiation. In this paper, we set out to investigate questions such as: Which time-stepping methods capture the solitons with more accuracy? How do they perform when computing highly-oscillatory solutions? How does their performance depend on the solution itself? Our strategy is as follows. Using a numerical inverse scattering transform (IST) method [5], we can accurately construct solutions of the Toda lattice for each $(n, t)$ without any time stepping, for arbitrarily large values of $t$, with high accuracy. Indeed, one can expect to maintain relative accuracy for large $t$ [23]. This opens the door to benchmarking time-stepping methods on solutions of different characters (oscillatory, has solitons, no solitons, etc.), without restricting oneself to those with exact formulae. Furthermore, it is reasonable to expect behavior of a numerical method for the Toda lattice to extend to other FPUT-type systems.

From a convergence (as time-step size tends to 0 ) and computational complexity point of views, comparison of numerical time-stepping methods is a well-trodden path, see for example, [14] for a detailed study in this direction, or the more recent survey article [7] by Butcher and the references therein. Using the numerical IST method, in this work we aim to add a new dimension to such studies.

The paper is organized as follows. In the remainder of this section we discuss specifics of the Toda lattice. In Section 2 we summarize the numerical IST procedure, give an overview of the numerical time-stepping methods used in this work, and present the explicit formulae for the initial data used in the numerical experiments. In Section 4, we compare performances of second order methods. In Section 5, we compare performances of higher order methods. We then draw some conclusions.

\footnotetext{
${ }^{1}$ Generic initial data for the Toda lattice gives rise to dispersive radiation.
} 
1.1. Properties of the Toda lattice. Complete integrability of the Toda lattice was proven by H. Flaschka [10] and S. V. Manakov [20] in 1974, independently and simultaneously, by realizing that the system possesses a Lax pair and (2) with $V(r)=V_{\mathrm{T}}(r)$ is in one-to-one correspondence with isospectral deformations of Jacobi (symmetric, tridiagonal with positive off-diagonal elements) matrices. Indeed, through the bijection

$$
a_{n}:=\frac{1}{2} e^{-\left(q_{n+1}-q_{n}\right) / 2}, \quad b_{n}:=-\frac{1}{2} p_{n},
$$

the equations of motion (1) Toda lattice take the form:

$$
\frac{\mathrm{d}}{\mathrm{d} t} a_{n}=a_{n}\left(b_{n+1}-b_{n}\right), \quad \frac{\mathrm{d}}{\mathrm{d} t} b_{n}=2\left(a_{n}^{2}-a_{n-1}^{2}\right), \quad n \in \mathbb{Z} .
$$

Defining the following second order linear difference operators $\mathbf{L}$ and $\mathbf{P}$ on the Hilbert space $\ell^{2}(\mathbb{Z})$ of square-summable sequences:

$$
\begin{aligned}
& (\mathbf{L} \phi)_{n}:=a_{n-1} \phi_{n-1}+b_{n} \phi_{n}+a_{n} \phi_{n+1} \\
& (\mathbf{P} \phi)_{n}:=-a_{n-1} \phi_{n-1}+a_{n} \phi_{n+1},
\end{aligned}
$$

it can be verified that (1) is equivalent to the Lax equation [18]

$$
\frac{\mathrm{d}}{\mathrm{d} t} \mathbf{L}=[\mathbf{P}, \mathbf{L}]:=\mathbf{P L}-\mathbf{L P} .
$$

The operators $(\mathbf{P}, \mathbf{L})$ are called a Lax pair and in the standard basis, $\mathbf{L}$ is a doubly-infinite Jacobi matrix. The Lax pair (8) constitutes the basis of to the IST method to solve the Cauchy initial value problem for (6) for sufficiently decaying initial data (see, for example, [2] for a recent survey of the IST for the Toda lattice).

A numerical IST method was recently developed by the authors [5] for the doubly-infinite Toda lattice. Implementations for other integrable systems can be found in $[23,27,28,30]$ and these are summarized in [29]. An implementation for the numerical IST method can be found at [4]. The method works, loosely speaking, by performing the following steps:

(1) Compute the spectral data: This involves solving the eigenvalue problem $\mathbf{L} \varphi=\lambda \varphi$ for for bounded eigenfunctions $\varphi$. Assuming $\left(a_{n}, b_{n}\right) \rightarrow(1 / 2,0)$ at an appropriately rapid rate as $|n| \rightarrow \infty$, the spectrum consists of the interval $[-1,1]$ and a finite number of simple eigenvalues in $\mathbb{R} \backslash[-1,1]$. One also computes, a function $R(z)$, defined on the spectrum, which is directly related to the spectral measure for $\mathbf{L}$.

(2) Solve the inverse problem: Once $R(z)$ is known, for each $(n, t)$ there exists a contour $\Gamma=$ $\Gamma(n, t) \subset \mathbb{C}$, a function $G(z ; n, t), G: \Gamma \rightarrow \mathbb{C}^{2 \times 2}$ and an integral equation

$$
\left(I-\frac{G}{2}\right) U+\mathcal{H}_{\Gamma}(G U)=G-I, \quad U: \Gamma \rightarrow \mathbb{C}^{2 \times 2} .
$$

Here $\mathcal{H}_{\Gamma}$ is the Hilbert transform over $\Gamma$ and $I$ is the identity matrix. This integral equation is solved for $U: \Gamma \rightarrow \mathbb{C}^{2 \times 2}$ using the framework of S. Olver [22] (see [21] for an implementation) and the solution of the Toda lattice can be obtained in terms of integrals of $U$. So, one can compute the map $(n, t) \mapsto U$ and hence $(n, t) \rightarrow\left(a_{n}(t), b_{n}(t)\right)$ without time stepping.

\section{Time-Stepping Methods}

In this section we describe the time-stepping methods used to produce the results shown in this paper. The first three methods described below are classical second-order methods and the 
following methods are higher-order methods. All of the methods are used to numerically compute the solution $y(T) \in \mathbb{R}^{N}$ of the Cauchy initial value problem for an autonomous differential equation

$$
\frac{d y}{d t}=f(y(t))
$$

with initial condition $y(0)=y^{0} \in \mathbb{R}^{N}$ at a final time $t=T$. We focus on classical methods to illustrate how one can use the numerical IST method to benchmark them.

Depending the nature of the scheme and the variables used, $f$ is an explicit, known function from $\mathbb{R}^{N}$ to $\mathbb{R}^{N}$ whose definition may differ among different methods (for example, in StörmerVerlet method). We truncate the spatial domain (the lattice $\mathbb{Z}$ ) to $S=\{-K, \ldots, 0, \ldots, K\}$, with the appropriate boundary conditions for the variables $(a, b)$ in $(6)$ or $(p, q)$ in $(2)$ and $K>0$ chosen large so that the $y(T)$ does not feel the effect of the boundary. Thus, $N=2 K+1$. In the descriptions that follow, $h>0$ denotes the time-step size. We use superscripts to denote the numerical iterates to avoid confusion with the indices of sequences: Given a computed solution $y^{k}$ at a time $t$, we denote the computed solution at time $t+h$ by $y^{k+1} . y_{n}^{k}$ is a scalar, the $n$-th element of the sequence $y^{k} \in \mathbb{R}^{N}$.

\subsection{Second-order Methods.}

2.1.1. Midpoint. We denote this second-order explicit method by midpoint. Given $y^{k}$, the algorithm to compute $y^{k+1}$ is as follows:

$$
\text { > } y^{k+1}=y^{k}+h f\left(y^{k}+\frac{1}{2} h f\left(y^{k}\right)\right)
$$

Unless otherwise stated, we integrate (6). When we integrate (2) instead we use the label midpointqp.

2.1.2. Second-order Störmer-Verlet. We denote this method by sv2symp. The Störmer-Verlet method is symplectic: It preserves the Hamiltonian (4) under exact arithmetic. We use the equations of motions (1) and set

$$
f_{p}(q):=\left(e^{-\left(q_{n}-q_{n-1}\right)}-e^{-\left(q_{n+1}-q_{n}\right)}\right)_{n \in \mathbb{Z}^{\prime}} \quad f_{q}(p):=\left(p_{n}\right)_{n \in \mathbb{Z}}
$$

to denote the right hand sides. Since the Hamiltonian for the Toda lattice is separable, the method becomes explicit. Given $\left(p^{k}, q^{k}\right)$, the algorithm to compute $\left(p^{k+1}, q^{k+1}\right)$ is as follows:

$$
\begin{aligned}
& \gg p^{k+1 / 2}=p^{k}+\frac{1}{2} h f_{p}\left(q^{k}\right) \\
& >q^{k+1}=q^{k}+h f_{q}\left(p^{k+1}\right) \\
& >p^{k+1}=p^{k+1}+\frac{1}{2} h f_{p}\left(q^{k+1}\right)
\end{aligned}
$$

\subsection{Fourth-order Methods.}

2.2.1. Fourth-order Adams-Bashforth. We denote this method by ab4. The Adams-Bashforth method is a linear explicit multi-step method. To compute the first three iterates we perform lower-step Adams-Bashforth methods successively:

$$
\begin{aligned}
& \text { > } y^{0}=y^{0} \\
& >y^{1}=y^{0}+h f\left(y^{0}\right) \\
& >y^{2}=y^{1}+\frac{1}{2} h\left(-f\left(y^{0}\right)+3 f\left(y^{1}\right)\right) \\
& >y^{3}=y^{2}+\frac{1}{12} h\left(5 f\left(y^{0}\right)-16 f\left(y^{1}\right)+23 f\left(y^{2}\right)\right)
\end{aligned}
$$

Then, given computed solutions $y^{k}, y^{k-1}, y^{k-2}, y^{k-3}, k \geq 3$ the algorithm to compute $y^{k+1}$ is as follows:

$$
\text { >> } y^{k+1}=y^{k}+\frac{1}{24} h\left(-9 f\left(y^{k-3}\right)+37 f\left(y^{k-2}\right)-59 f\left(y^{k-1}\right)+55 f\left(y^{k}\right)\right)
$$


2.2.2. Fourth-order Runge-Kutta. We denote this method by rk4. The Runge-Kutta method is a an explicit single-step method. Given the computed solution $y^{k}$, the algorithm $[17,24]$ to compute $y^{k+1}$ is as follows:

$$
\begin{aligned}
& >s_{1}=h f\left(y^{k}\right) \\
& >s_{2}=h f\left(y^{0}+\frac{1}{2} s_{1}\right) \\
& >s_{3}=h f\left(y^{0}+\frac{1}{2} s_{2}\right) \\
& >s_{4}=h f\left(y^{0}+s_{3}\right) \\
& >y^{k+1}=y^{k}+\frac{1}{6} h\left(s_{1}+2 s_{2}+2 s_{3}+s_{4}\right)
\end{aligned}
$$

Unless otherwise stated, we integrate (6). When we integrate (2) we use the label rk4qp.

2.2.3. Four-Five-order Runge-Kutta-Fehlberg. We denote this method by rkf 45 . This method is fourth order method but with only a single extra computation at each step the local error can be controlled by a fifth order method. This feature [8] is very practical for implementing adaptivestep-size methods. We do not implement any adaptive methods. Given the computed solution $y^{k}$, the algorithm $[8,12]$ to compute $y^{k+1}$ is as follows:

$$
\begin{aligned}
& >s_{1}=h f(y) \\
& >s_{2}=f\left(y+h b_{21} s_{1}\right) \\
& >s_{3}=f\left(y+h\left(b_{31} s_{1}+b_{32} s_{2}\right)\right) \\
& >s_{4}=f\left(y+h\left(b_{41} s_{1}+b_{42} s_{2}+b_{43} s_{3}\right)\right) \\
& >s_{5}=f\left(y+h\left(b_{51} s_{1}+b_{52} s_{2}+b_{53} s_{3}+b_{54} s_{4}\right)\right) \\
& >s_{6}=f\left(y+h\left(b_{61} s_{1}+b_{62} s_{2}+b_{63} s_{3}+b_{64} s_{4}+b_{65} s_{5}\right)\right) \\
& >y^{k+1}=y^{k}+h\left(c_{1} s_{1}+c_{2} s_{2}+c_{3} s_{3}+c_{4} s_{4}+c_{5} s_{5}+c_{6} s_{6}\right)
\end{aligned}
$$

For the constants $b_{i j}$ and $c_{j}$, see the Butcher tableau provided in the Appendix A. See [6] for a survey article by Butcher on Runge-Kutta methods.

Remark 2.1. For a detailed study of these methods and more, the reader may consult to textbooks [12] or [19], for example.

\section{INITIAL DATA CONSIDERED IN NUMERICAL EXPERIMENTS}

We now present the types of initial data (ID) used in the numerical experiments underlying the results presented in this paper.

3.0.1. Purely dispersive (NoS). We chose ID that gives rise to pure radiation: There are no solitons since the discrete spectrum of $\mathbf{L}$ is empty. The ID is given by

$$
a_{n}=\frac{1}{2}-\frac{1}{4} e^{-n^{2}}, \quad b_{n}=\frac{1}{10} \operatorname{sech}(n), \quad n \in \mathbb{Z} .
$$

The solution at $t=1000$ is shown in Figure 2 .

3.0.2. Pure 1-soliton (PureS). We chose a pure 1-soliton solution of the Toda lattice at time $t=0$. This ID is given by

$$
\begin{aligned}
& a_{n}=1-\frac{1}{2} \frac{\sqrt{\left(1+e^{-2 \kappa(n-1)}\right)\left(1+e^{-2 \kappa(n+1)}\right)}}{1+e^{-2 \kappa n}}, \\
& b_{n}=\frac{e^{-\kappa}-e^{\kappa}}{2}\left(\frac{e^{-2 \kappa n}}{1+e^{-2 \kappa n}}-\frac{e^{-2 \kappa(n-1)}}{1+e^{-2 \kappa(n-1)}}\right), \quad \kappa=0.4, \quad n \in \mathbb{Z} .
\end{aligned}
$$

The solution at $t=1000$ is shown in Figure 3. Notice that this is the only solution we consider without dispersive radiation. 

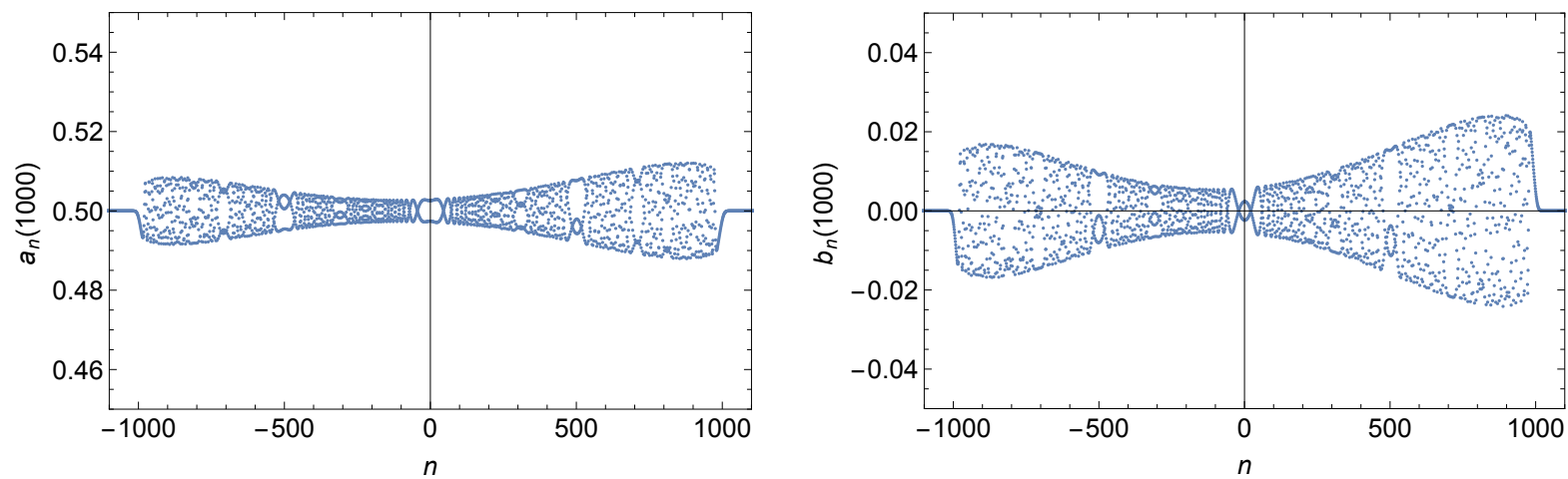

FIGURE 2. The solution of the Toda lattice with NoS ID at $t=1000$.
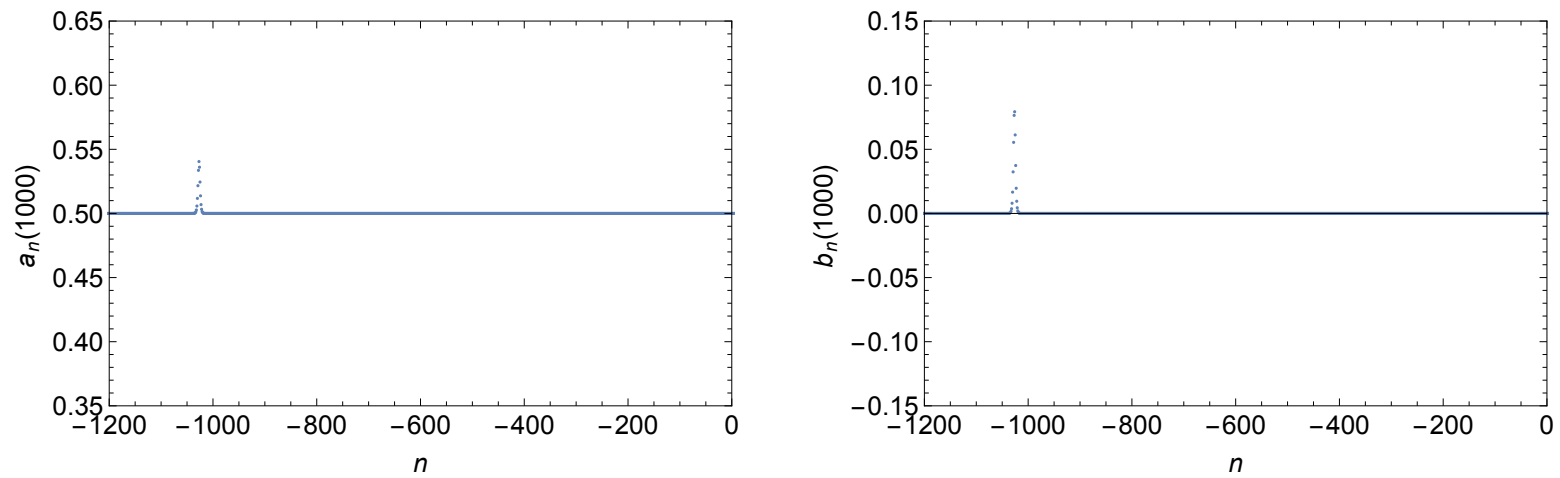

FIGURE 3. The solution of the Toda lattice with PureS ID at $t=1000$.

3.0.3. 2 solitons (double). A choice of ID that gives rise to 2 solitons and radiation is

$$
a_{n}=\frac{1}{2}+\frac{4}{5} n e^{-n^{2}}, \quad b_{n}=\frac{1}{10} \operatorname{sech}(n), \quad n \in \mathbb{Z} .
$$

The solution at $t=1000$ is shown in Figure 4 .

3.0.4. 4 solitons (quad). ID that gives rise to 4 solitons and radiation is given by

$$
a_{n}=\left|\frac{1}{2}-n e^{-n^{2}+n}\right|, \quad b_{n}=n \operatorname{sech}(n), \quad n \in \mathbb{Z} .
$$

The solution at $t=1000$ is shown in Figure 5 .

3.0.5. Dirac $\delta$-type (dirac). Dirac- $\delta$-type ID that leads to 1 soliton and a radiating tail that is highly oscillatory is simply given by

$$
a_{n}=\frac{1}{2}, \quad b_{n}=\left\{\begin{array}{ll}
4, & n=0 \\
0, & \text { otherwise }
\end{array}, \quad n \in \mathbb{Z} .\right.
$$

The solution at $t=1000$ is shown in Figure 6. 



FIGURE 4. The solution of the Toda lattice with double ID at $t=1000$.


FIGURE 5. The solution of the Toda lattice with quad ID at $t=1000$.
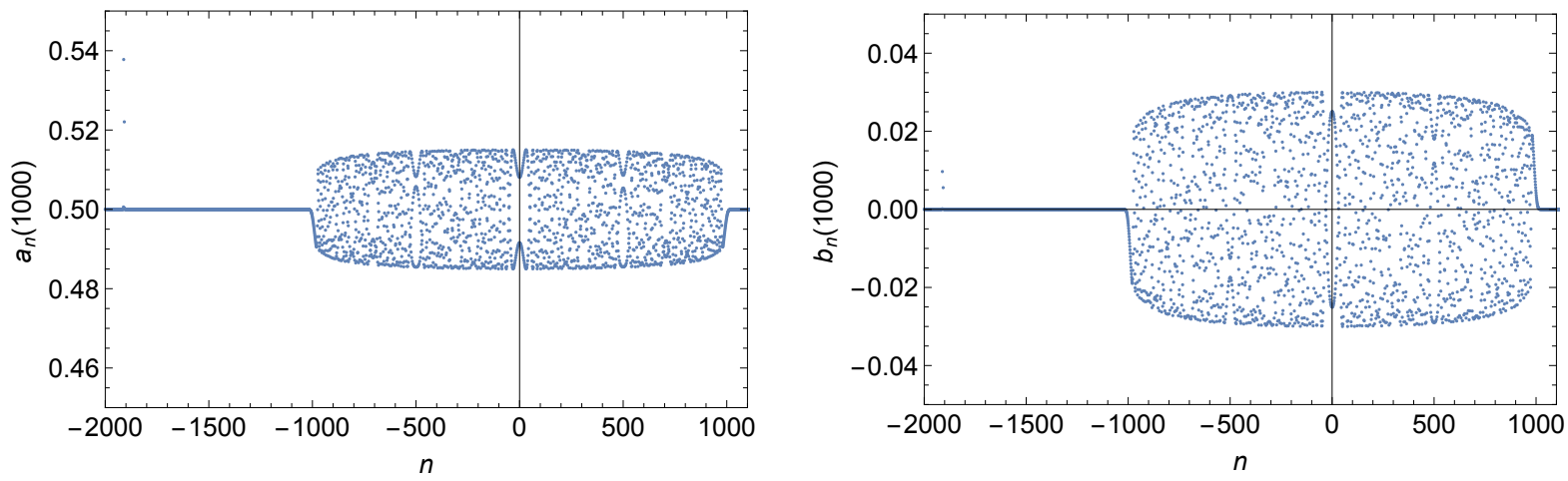

FIGURE 6. The solution of the Toda lattice with dirac ID at $t=1000$.

\section{Comparison of SeCond-Order Methods}

In order to compare our methods we take the following approach. We choose three time steps $d T=0.01,0.001,0.0001$, three final times $T=1000,2000,5000$, and two regions (dispersive and 

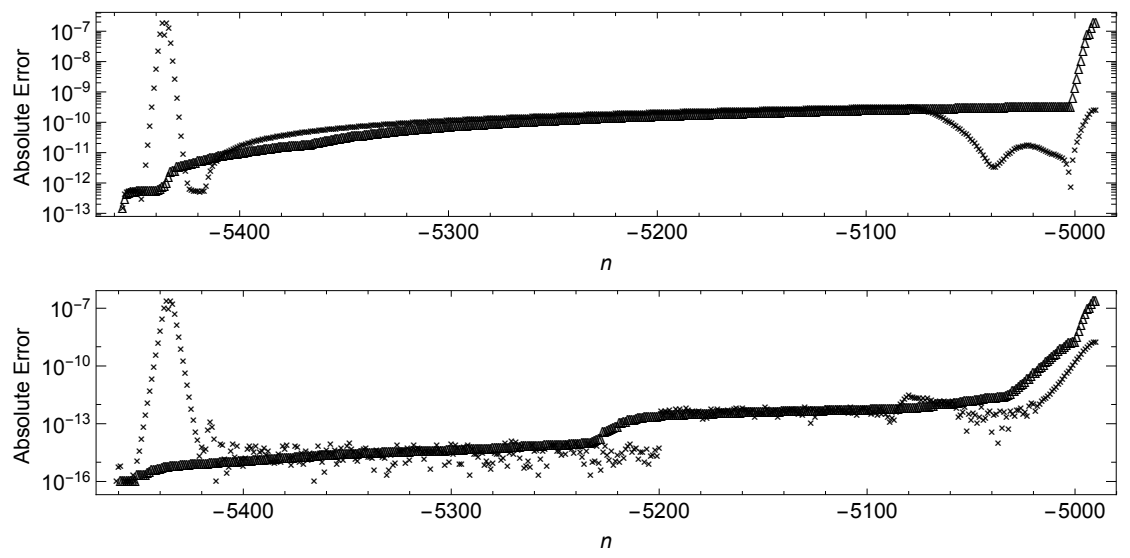

FIGURE 7. Absolute errors in the soliton region, for quad ID, $d T=0.0001$ with the ab4 (top) and sv2symp (bottom) methods. The $\times$ symbols represent the actual absolute errors as a function of $n$ and the $\Delta$ symbols are the errors sorted in increasing order.

soliton regions, for only $n<0$ ) and examine the relative errors made in approximating the solution of the Toda lattice at the final times for every choice of ID and every choice of time-stepping method. In accordance with the asymptotic analysis $[5,15,16]$, the soliton region is effectively $\mathbb{Z} \backslash[-T, T]$. And so, the soliton region for this work is $[-(s+100) T,-T]$ where $s$ is the speed of the fastest moving soliton. This can be computed from the spectrum of $\mathbf{L}$ [5]. The dispersive region is $[-c T, c T]$ for $0<c<1$. So, we fix our dispersive region as $[-T / 2-50,-T / 2+50]$. We always take the solution computed with the numerical IST method to be our "true" solution. A significant benefit of the numerical IST method is that the solution at each $(n, t)$ can be computed independently of all others. Thus to compute the "true" solution we only need to compute on the intervals $[-(s+100) T,-T]$ and $[-T / 2-50,-T / 2+50]$ and we can refrain from computing the entire solution profile.

To define the relative error measure we use, consider the plot of errors in the soliton region, for quad ID, $d T=0.0001$ with the ab4 and sv2symp methods as shown in Figure 7 . If one uses an $\infty$-norm (or max norm) measurement (see the $\times$ symbols in Figure 7 ), one might conclude that the errors are comparable. What this fails to account for is that the sv2symp method has fewer errors that are near the maximum error when compared with the ab4 method. To account for this we define the sorted norm on $\mathbb{R}^{n}$ for $0<d<1$ by

$$
\|x\|_{\text {sort }, d}=\left\|\left(y_{1}, y_{2}, \ldots, y_{\lceil d n\rceil}\right)^{T}\right\|_{2}, \quad y=\operatorname{sort}(|x|) .
$$

Here the function $\operatorname{sort}(\cdot)$ sorts the positive vector $|x|$ in decreasing order, and the sorted norm takes the $\ell^{2}$ norm of the largest $\sim d n$ entries of the vector $|x|$. In all our computation we take $d=0.1$, taking $10 \%$ of the entries. This is a hybrid of the $\ell^{2}$-norm and the $\infty$-norm Then the error of a vector $x$ relative to a vector $y$ with background $c$ is given by

$$
\operatorname{rel}_{y, c}(x)=\frac{\|x-y\|_{\text {sort }, d}}{\|c-y\|_{\text {sort }, d}} .
$$

We introduce the background $c$ because for fixed $n, a_{n}(t) \rightarrow 1 / 2$ as $t \rightarrow \infty$ while $b_{n}(t) \rightarrow 0$ as $t \rightarrow \infty$. We want to approximate $a_{n}(t)-1 / 2$ and $b_{n}(t)$.

Remark 4.1. For PureS ID in the dispersive region, the solution is, to machine precision, zero. A relative error metric here does not make sense and we use absolute error $\|x-y\|_{\text {sort }, s}$. 



FIGURE 8. Relative errors in the soliton region for the second-order time-stepping methods (midpoint $(X)$, midpointqp $(\Delta)$, sv2symp $(\square)$ ) at $T=1000$ plotted versus $d T$ for three choices of time step for three different choices of ID.
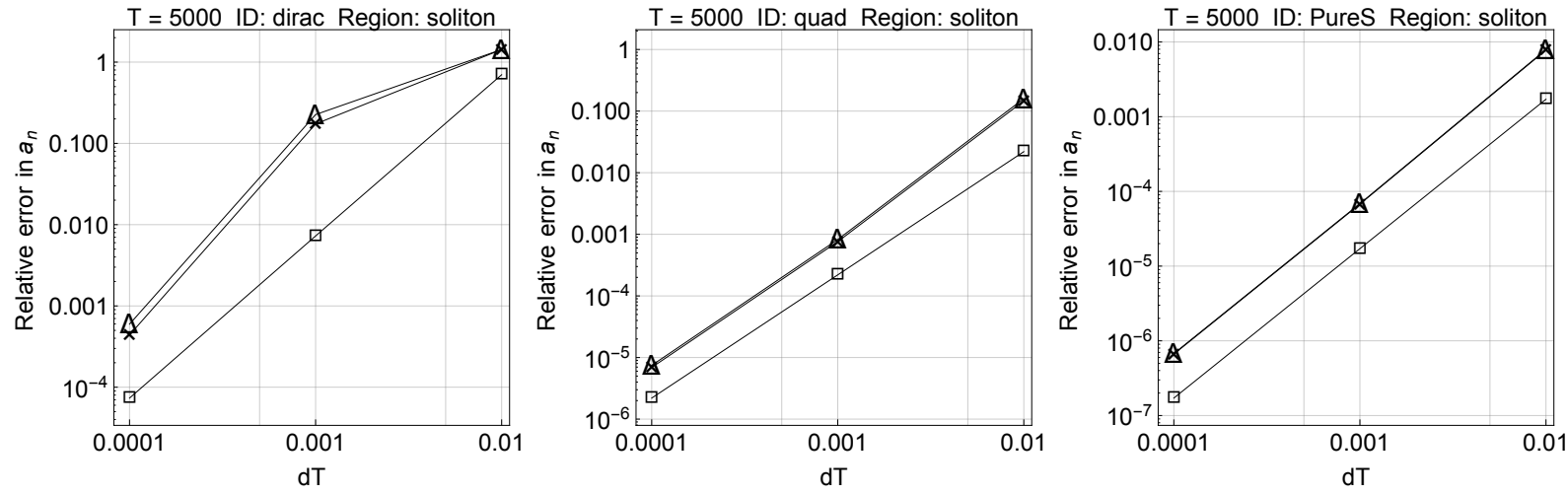

FIGURE 9. Relative errors in the soliton region for the second-order time-stepping methods (midpoint $(\times)$, midpointqp $(\Delta)$, sv2symp $(\square)$ ) at $T=5000$ plotted versus $d T$ for three choices of time step for three different choices of ID.

4.1. Soliton Region. We first consider the errors $\operatorname{rel}_{y, 1 / 2}(x)$ made in the approximation of the solution $a_{n}(T)$ of the Toda lattice in the soliton region $[-(s+100) T,-T]$ at time $T$. Here $y$ is chosen to be the reference solution obtained by the numerical IST method. In each panel of Figure 8 we plot the relative error of the computed solution plotted versus $d T$. In all panels sv2symp out performs the other methods.

4.2. Dispersive Region. We now consider the errors made in the approximation of the solution $a_{n}(T)$ of the Toda lattice in the dispersive region region $[-T / 2-50,-T / 2+50]$ at time $T$. We can make an important point with Figure 10 and 11. If one could only work with the pure soliton solution (right panel in the figures), that person might conclude that the midpointqp for small enough time step performs as well as sv2symp away from the soliton. This is true for the PureS ID, but not for the other ID. This illustrates why having accurate solutions with dispersive tails to compare against is important.

\section{COMPARISON OF FOURTh-Order MEthods}

We now move to the comparison of the fourth-order methods listed above. We use the same relative error metric $\operatorname{rel}_{y, 1 / 2}(x)$ as described in (15), where the reference solution $y$ is computed with the numerical IST method. 

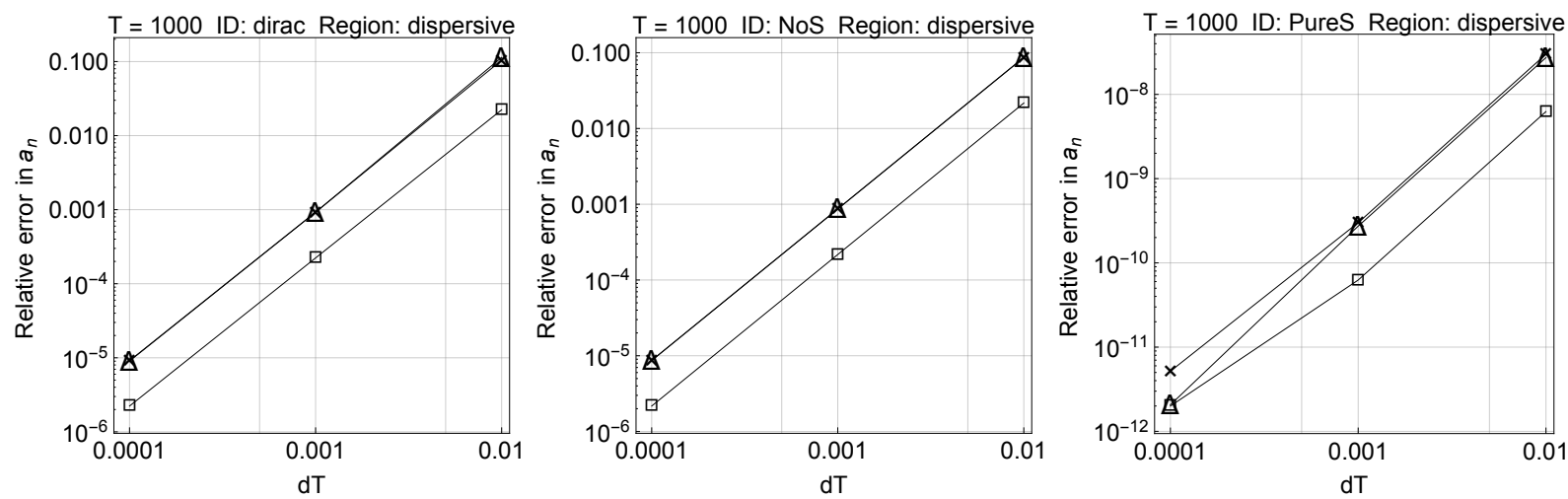

FIGURE 10. Relative errors in the dispersive region for the second-order timestepping methods (midpoint $(x)$, midpointqp $(\Delta)$, sv2symp $(\square)$ ) at $T=1000$ plotted versus $d T$ for three choices of time step for three different choices of ID.
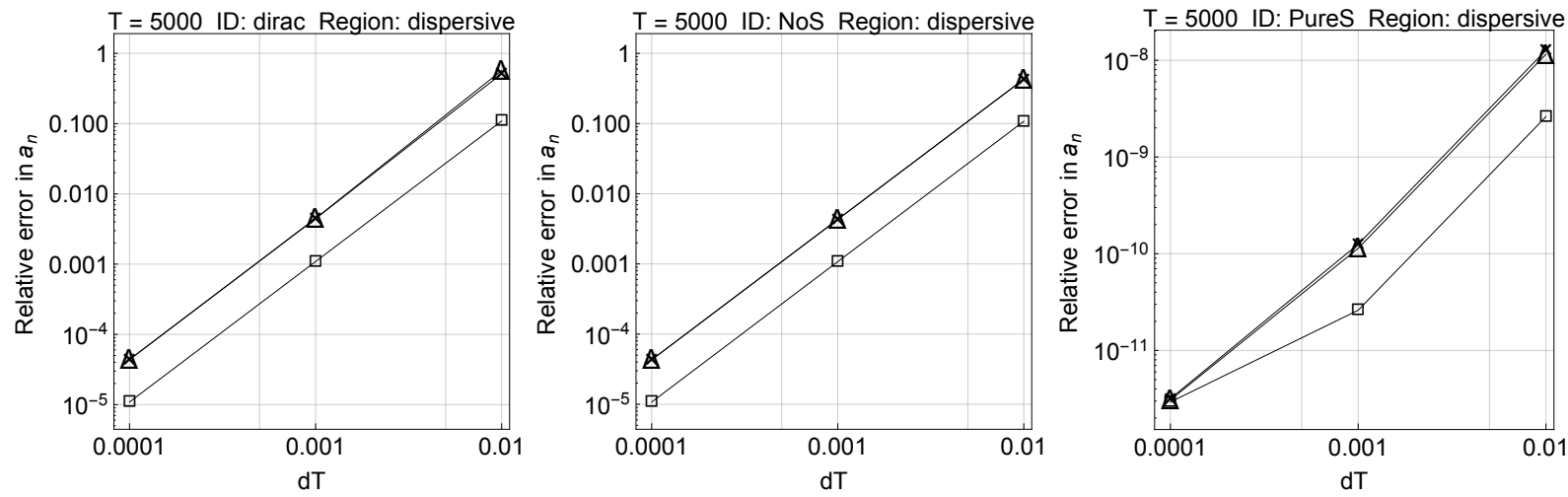

FIGURE 11. Relative errors in the dispersive region for the second-order timestepping methods (midpoint $(x)$, midpointqp $(\Delta)$, sv2symp $(\square)$ ) at $T=5000$ plotted versus $d T$ for three choices of time step for three different choices of ID.

5.1. Soliton Region. In each of the panels of Figures 12 and 13 we plot the relative error of the computed approximation of $a_{n}(T)$ plotted versus $d T$ for fourth-order methods in the soliton region. We can see that we are operating near the maximum accuracy of these methods as the relative error can increase as $d T$ decreases. We see that ab4 under-performs and rk4qp is almost always the method of choice in this region. But it is important to note that the relative error encountered for the PureS ID is less than that encountered for the other ID. This points again to the need for a wide class of test solutions.

5.2. Dispersive Region. In each of the panels of Figures 14 and 15 we plot the relative error of the computed approximation of $a_{n}(T)$ plotted versus $d T$ for fourth-order method in the dispersive region. We can again see that we are operating near the maximum accuracy. We see that ab4 under-performs but not as severely as in the soliton region and rk4qp is still almost always the method of choice in this region, at least for small time steps. Again, the PureS ID gives smaller errors (recall we can only measure the absolute error for PureS ID in the dispersive region) than the other choices of ID, illustrating the importance of being able to compute these solutions accurately with the numerical IST method. 

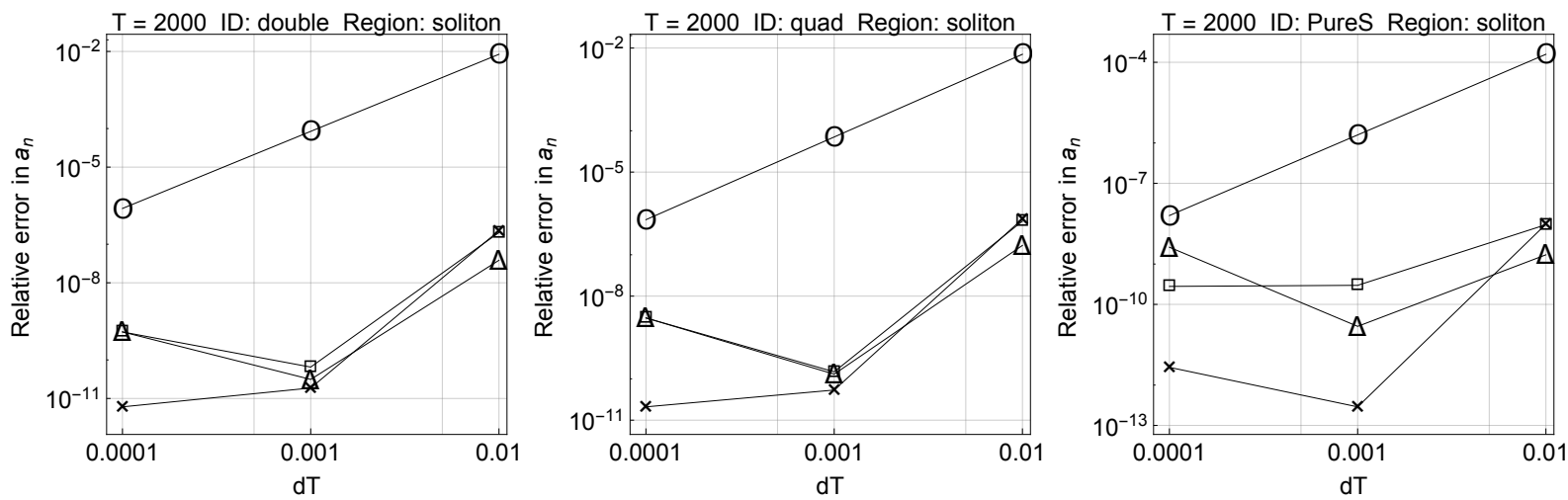

FIGURE 12. Relative errors in the soliton region for the fourth-order time-stepping methods $(r k 4(\square), \operatorname{rk} 4 q p(\times), \operatorname{rkf} 45(\Delta)$, ab4 $(\bigcirc))$ at $T=2000$ plotted versus $d T$ for three choices of time step for three different choices of ID.
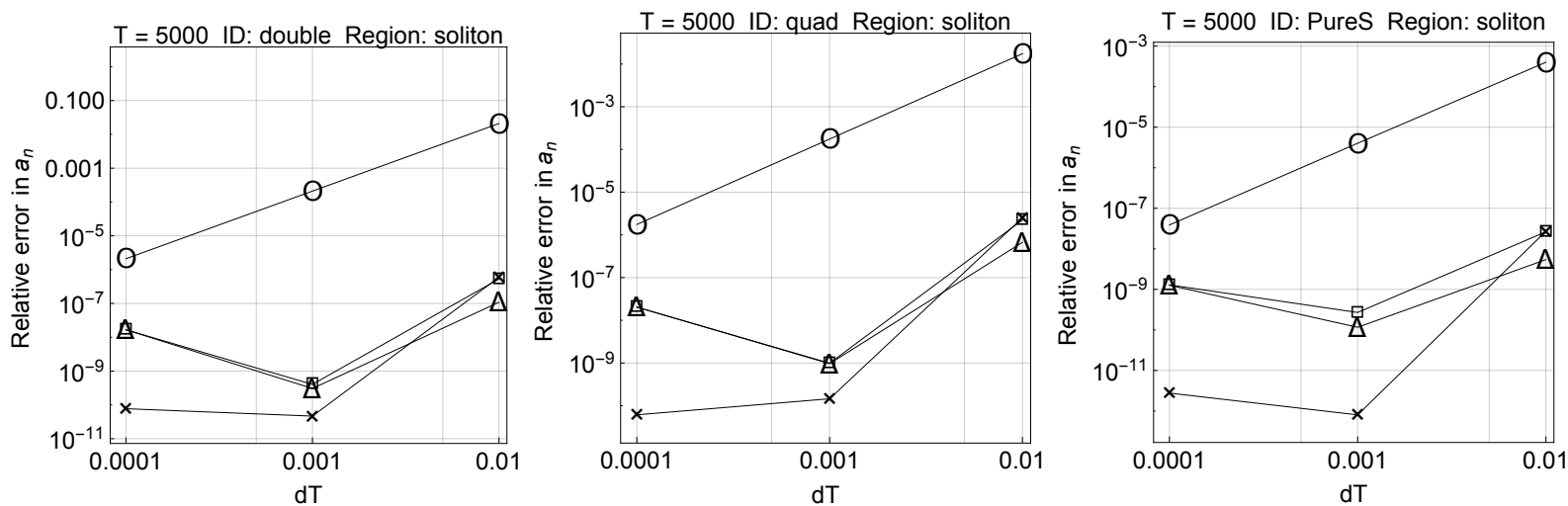

FIGURE 13. Relative errors in the soliton region for the fourth-order time-stepping methods $(r k 4(\square), \operatorname{rk} 4 q p(\times), \operatorname{rkf} 45(\Delta)$, ab4 $(\bigcirc))$ at $T=5000$ plotted versus $d T$ for three choices of time step for three different choices of ID.
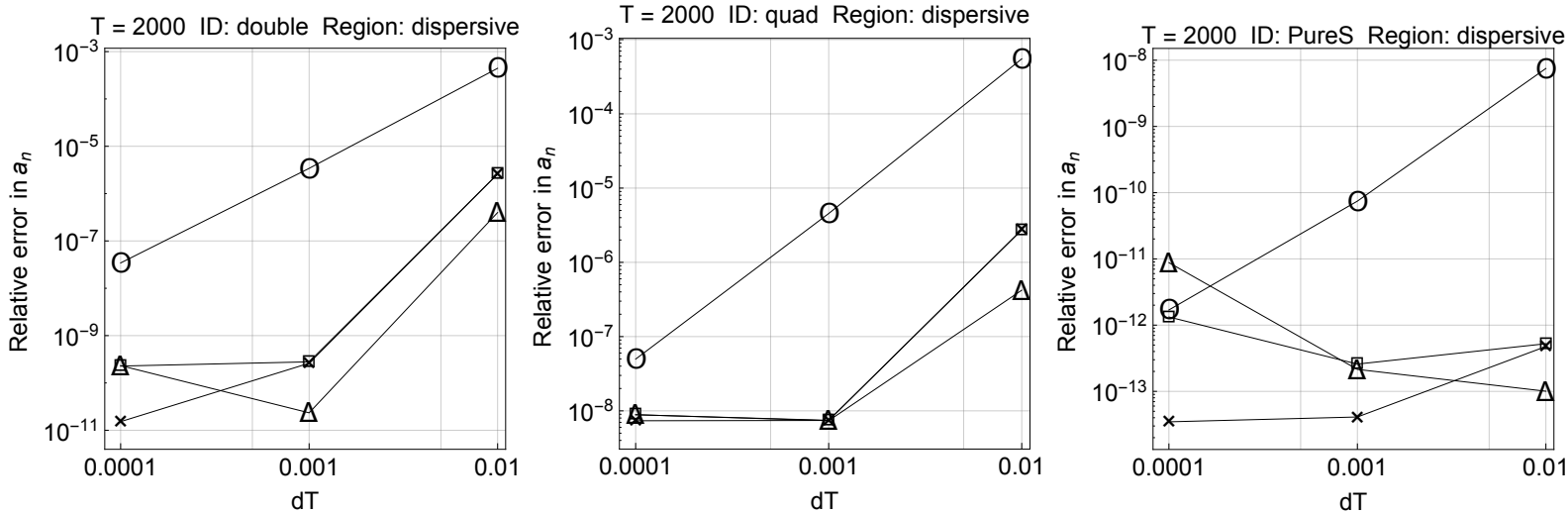

FIGURE 14. Relative errors in the dispersive region for the fourth-order timestepping methods $(r k 4(\square), \operatorname{rk4qp}(\times), \operatorname{rkf} 45(\Delta)$, ab4 $(\bigcirc))$ at $T=2000$ plotted versus $d T$ for three choices of time step for three different choices of ID. 

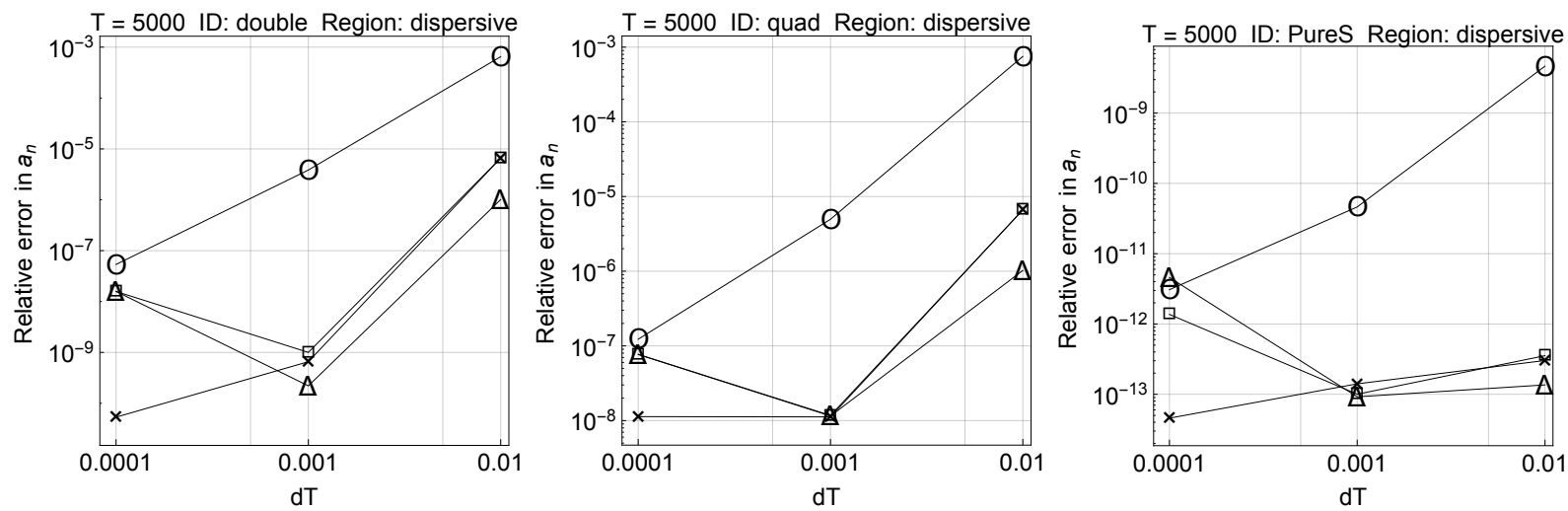

FIGURE 15. Relative errors in the dispersive region for the fourth-order timestepping methods $(r k 4(\square), \operatorname{rk} 4 q p(\times), \operatorname{rkf} 45(\Delta), a b 4(\bigcirc))$ at $T=5000$ plotted versus $d T$ for three choices of time step for three different choices of ID.

\section{CONCLUSIONS}

We have used the numerical IST method outlined in [5] to benchmark classical time-stepping routines on the Toda lattice. It is reasonable, especially following the perturbation work in [3], that such benchmarks will hold for other lattice equations - not just the Toda lattice and not just integrable lattices. The method rk4qp appears to be the method of choices while the symplectic method sv2symp performs surprisingly well given that it is only second-order accurate.

We have illustrated that having a wider class of methods allows one to detect deeper differences in methods, and analyze the accumulation of round-off error while getting a handle on the maximum accuracy of a method. We have also shown that benchmarking a method on a pure soliton initial condition can lead one to overestimate the maximum accuracy of the method. As we consider nonlinear lattices, both the lattice equation and the choice of ID matter in the performance of a given method.

In Appendix B we give a complete listing of relative errors for both $a_{n}$ and $b_{n}$. In [1] we have made available our data for the reference solutions computed with the numerical IST to allow others to benchmark their time-stepping routines.

\section{ACKNOWLEDGMENTS}

D.B. is thankful for partial support from the National Science Foundation through the grant NSF-DMS-1513054.

\section{Appendix A. Butcher TABleAu FOR FeHLBERG'S RKF45}

We provide the Butcher tableau for the constants used in the RKF45 method in this paper [8].

\begin{tabular}{c|cccccc}
$j$ & 1 & 2 & 3 & 4 & 5 & 6 \\
\hline$b_{2 j}$ & $\frac{1}{4}$ & & & & & \\
$b_{3 j}$ & $\frac{3}{32}$ & $\frac{9}{32}$ & & & & \\
$b_{4 j}$ & $\frac{1932}{2197}$ & $\frac{-7200}{2197}$ & $\frac{7296}{2197}$ & & & \\
$b_{5 j}$ & $\frac{439}{216}$ & -8 & $\frac{3680}{513}$ & $\frac{-845}{4104}$ & & \\
$b_{6 j}$ & $\frac{-8}{27}$ & 2 & $\frac{-3544}{2565}$ & $\frac{1859}{4104}$ & $\frac{-11}{40}$ & \\
\hline$c_{j}$ & $\frac{25}{216}$ & 0 & $\frac{1408}{2565}$ & $\frac{2197}{4104}$ & $\frac{-1}{5}$ & 0
\end{tabular}




\section{APPENDiX B. DATA FOR THE METHODS}

In the tables below, we display the data for the methods discussed in the body of the paper. In each table we display the performance of 3-4 methods, run until $T=1000,2000,3000$ each with three different time steps. Each table is associated to a choice of initial data (dirac, double, NoS, PureS, quad) and a region (soliton region $=$ Sol ., dispersive region = Disp.).

B.1. Second order - errors for $a_{n}(t)$.

\begin{tabular}{|c|c|c|c|c|c|c|c|c|c|}
\hline quad/Sol. & & $\mathrm{T}=1000$ & & & $T=2000$ & & & $\mathrm{~T}=5000$ & \\
\hline $\mathrm{dT}$ & 0.01 & 0.001 & 0.0001 & 0.01 & 0.001 & 0.0001 & 0.01 & 0.001 & 0.0001 \\
\hline sv2symp & $4.499 \times 10^{-3}$ & $4.499 \times 10^{-5}$ & $4.499 \times 10^{-7}$ & $8.914 \times 10^{-3}$ & $8.912 \times 10^{-5}$ & $8.912 \times 10^{-7}$ & $2.195 \times 10^{-2}$ & $2.196 \times 10^{-4}$ & $2.197 \times 10^{-6}$ \\
\hline midpoint & $1.834 \times 10^{-2}$ & $1.441 \times 10^{-4}$ & $1.402 \times 10^{-6}$ & $4.222 \times 10^{-2}$ & $2.913 \times 10^{-4}$ & $2.782 \times 10^{-6}$ & $1.463 \times 10^{-1}$ & $7.608 \times 10^{-4}$ & $6.898 \times 10^{-6}$ \\
\hline midpointqp & $2.004 \times 10^{-2}$ & $1.561 \times 10^{-4}$ & $1.516 \times 10^{-6}$ & $4.608 \times 10^{-2}$ & $3.153 \times 10^{-4}$ & $3.008 \times 10^{-6}$ & $1.593 \times 10^{-1}$ & $8.234 \times 10^{-4}$ & $7.456 \times 10^{-6}$ \\
\hline quad/Disp. & & $\mathrm{T}=1000$ & & & $\mathrm{~T}=2000$ & & & $\mathrm{~T}=5000$ & \\
\hline $\mathrm{dT}$ & 0.01 & 0.001 & 0.0001 & 0.01 & 0.001 & 0.0001 & 0.01 & 0.001 & 0.0001 \\
\hline sv2symp & $2.218 \times 10^{-2}$ & $2.217 \times 10^{-4}$ & $2.218 \times 10^{-6}$ & $4.43 \times 10^{-2}$ & $4.429 \times 10^{-4}$ & $4.428 \times 10^{-6}$ & $1.084 \times 10^{-1}$ & $1.084 \times 10^{-3}$ & $1.084 \times 10^{-5}$ \\
\hline midpoint & $8.916 \times 10^{-2}$ & $8.891 \times 10^{-4}$ & $8.891 \times 10^{-6}$ & $1.781 \times 10^{-1}$ & $1.776 \times 10^{-3}$ & $1.775 \times 10^{-5}$ & $4.347 \times 10^{-1}$ & $4.341 \times 10^{-3}$ & $4.333 \times 10^{-5}$ \\
\hline midpointqp & $8.954 \times 10^{-2}$ & $8.896 \times 10^{-4}$ & $8.892 \times 10^{-6}$ & $1.788 \times 10^{-1}$ & $1.777 \times 10^{-3}$ & $1.776 \times 10^{-5}$ & $4.364 \times 10^{-1}$ & $4.343 \times 10^{-3}$ & $4.34 \times 10^{-5}$ \\
\hline dirac/Sol. & & $\mathrm{T}=1000$ & & & $T=2000$ & & & $\mathrm{~T}=5000$ & \\
\hline $\mathrm{dT}$ & 0.01 & 0.001 & 0.0001 & 0.01 & 0.001 & 0.0001 & 0.01 & 0.001 & 0.0001 \\
\hline sv2symp & $2.805 \times 10^{-1}$ & $2.937 \times 10^{-3}$ & $2.938 \times 10^{-5}$ & $3.859 \times 10^{-1}$ & $2.729 \times 10^{-3}$ & $2.715 \times 10^{-5}$ & $6.994 \times 10^{-1}$ & $7.258 \times 10^{-3}$ & $7.347 \times 10^{-5}$ \\
\hline midpoint & 1.622 & $2.808 \times 10^{-2}$ & $9.098 \times 10^{-5}$ & 1.451 & $4.956 \times 10^{-2}$ & $1.036 \times 10^{-4}$ & 1.428 & $1.734 \times 10^{-1}$ & $4.393 \times 10^{-4}$ \\
\hline midpointqp & 1.634 & $3.753 \times 10^{-2}$ & $1.285 \times 10^{-4}$ & 1.322 & $6.718 \times 10^{-2}$ & $1.443 \times 10^{-4}$ & 1.43 & $2.261 \times 10^{-1}$ & $5.969 \times 10^{-4}$ \\
\hline dirac/Disp. & & $T=1000$ & & & $\mathrm{~T}=2000$ & & & $\mathrm{~T}=5000$ & \\
\hline $\mathrm{dT}$ & 0.01 & 0.001 & 0.0001 & 0.01 & 0.001 & 0.0001 & 0.01 & 0.001 & 0.0001 \\
\hline sv2symp & $2.229 \times 10^{-2}$ & $2.23 \times 10^{-4}$ & $2.23 \times 10^{-6}$ & $4.381 \times 10^{-2}$ & $4.383 \times 10^{-4}$ & $4.383 \times 10^{-6}$ & $1.091 \times 10^{-1}$ & $1.089 \times 10^{-3}$ & $1.089 \times 10^{-5}$ \\
\hline midpoint & $1.044 \times 10^{-1}$ & $9.254 \times 10^{-4}$ & $9.138 \times 10^{-6}$ & $2.04 \times 10^{-1}$ & $1.801 \times 10^{-3}$ & $1.778 \times 10^{-5}$ & $5.126 \times 10^{-1}$ & $4.444 \times 10^{-3}$ & $4.386 \times 10^{-5}$ \\
\hline midpointqp & $1.137 \times 10^{-1}$ & $9.241 \times 10^{-4}$ & $9.033 \times 10^{-6}$ & $2.242 \times 10^{-1}$ & $1.809 \times 10^{-3}$ & $1.768 \times 10^{-5}$ & $5.709 \times 10^{-1}$ & $4.481 \times 10^{-3}$ & $4.378 \times 10^{-5}$ \\
\hline double/Sol. & & $T=1000$ & & & $T=2000$ & & & $\mathrm{~T}=5000$ & \\
\hline $\mathrm{dT}$ & 0.01 & 0.001 & 0.0001 & 0.01 & 0.001 & 0.0001 & 0.01 & 0.001 & 0.0001 \\
\hline sv2symp & $1.724 \times 10^{-3}$ & $1.723 \times 10^{-5}$ & $1.723 \times 10^{-7}$ & $3.388 \times 10^{-3}$ & $3.387 \times 10^{-5}$ & $3.387 \times 10^{-7}$ & $8.378 \times 10^{-3}$ & $8.377 \times 10^{-5}$ & $8.378 \times 10^{-7}$ \\
\hline midpoint & $7.759 \times 10^{-3}$ & $7.098 \times 10^{-5}$ & $7.03 \times 10^{-7}$ & $1.588 \times 10^{-2}$ & $1.404 \times 10^{-4}$ & $1.385 \times 10^{-6}$ & $4.366 \times 10^{-2}$ & $3.52 \times 10^{-4}$ & $3.434 \times 10^{-6}$ \\
\hline midpointqp & $8.85 \times 10^{-3}$ & $8.088 \times 10^{-5}$ & $8.013 \times 10^{-7}$ & $1.804 \times 10^{-2}$ & $1.598 \times 10^{-4}$ & $1.577 \times 10^{-6}$ & $4.919 \times 10^{-2}$ & $3.999 \times 10^{-4}$ & $3.907 \times 10^{-6}$ \\
\hline double/Disp. & & $\mathrm{T}=1000$ & & & $T=2000$ & & & $\mathrm{~T}=5000$ & \\
\hline $\mathrm{dT}$ & 0.01 & 0.001 & 0.0001 & 0.01 & 0.001 & 0.0001 & 0.01 & 0.001 & 0.0001 \\
\hline sv2symp & $2.193 \times 10^{-2}$ & $2.193 \times 10^{-4}$ & $2.193 \times 10^{-6}$ & $4.328 \times 10^{-2}$ & $4.329 \times 10^{-4}$ & $4.329 \times 10^{-6}$ & $1.099 \times 10^{-1}$ & $1.099 \times 10^{-3}$ & $1.099 \times 10^{-5}$ \\
\hline midpoint & $8.748 \times 10^{-2}$ & $8.763 \times 10^{-4}$ & $8.765 \times 10^{-6}$ & $1.725 \times 10^{-1}$ & $1.73 \times 10^{-3}$ & $1.731 \times 10^{-5}$ & $4.369 \times 10^{-1}$ & $4.393 \times 10^{-3}$ & $4.392 \times 10^{-5}$ \\
\hline midpointqp & $8.768 \times 10^{-2}$ & $8.765 \times 10^{-4}$ & $8.765 \times 10^{-6}$ & $1.729 \times 10^{-1}$ & $1.731 \times 10^{-3}$ & $1.731 \times 10^{-5}$ & $4.378 \times 10^{-1}$ & $4.394 \times 10^{-3}$ & $4.394 \times 10^{-5}$ \\
\hline
\end{tabular}




\begin{tabular}{|c|c|c|c|c|c|c|c|c|c|}
\hline NoS/Sol. & & $T=1000$ & & & $\mathrm{~T}=2000$ & & & $\mathrm{~T}=5000$ & \\
\hline $\mathrm{dT}$ & 0.01 & 0.001 & 0.0001 & 0.01 & 0.001 & 0.0001 & 0.01 & 0.001 & 0.0001 \\
\hline sv2symp & $1.762 \times 10^{-5}$ & $1.762 \times 10^{-7}$ & $1.762 \times 10^{-9}$ & $2.428 \times 10^{-5}$ & $2.427 \times 10^{-7}$ & $2.427 \times 10^{-9}$ & $3.18 \times 10^{-5}$ & $3.18 \times 10^{-7}$ & $3.18 \times 10^{-9}$ \\
\hline midpoint & $7.551 \times 10^{-5}$ & $7.512 \times 10^{-7}$ & $7.549 \times 10^{-9}$ & $1.031 \times 10^{-4}$ & $1.026 \times 10^{-6}$ & $1.034 \times 10^{-8}$ & $1.332 \times 10^{-4}$ & $1.327 \times 10^{-6}$ & $1.418 \times 10^{-8}$ \\
\hline midpointqp & $7.82 \times 10^{-5}$ & $7.767 \times 10^{-7}$ & $7.762 \times 10^{-9}$ & $1.06 \times 10^{-4}$ & $1.054 \times 10^{-6}$ & $1.053 \times 10^{-8}$ & $1.358 \times 10^{-4}$ & $1.352 \times 10^{-6}$ & $1.352 \times 10^{-8}$ \\
\hline NoS/Disp. & & $\mathrm{T}=1000$ & & & $T=2000$ & & & $\mathrm{~T}=5000$ & \\
\hline $\mathrm{dT}$ & 0.01 & 0.001 & 0.0001 & 0.01 & 0.001 & 0.0001 & 0.01 & 0.001 & 0.0001 \\
\hline sv2symp & $2.167 \times 10^{-2}$ & $2.167 \times 10^{-4}$ & $2.167 \times 10^{-6}$ & $4.304 \times 10^{-2}$ & $4.303 \times 10^{-4}$ & $4.303 \times 10^{-6}$ & $1.077 \times 10^{-1}$ & $1.077 \times 10^{-3}$ & $1.077 \times 10^{-5}$ \\
\hline midpoint & $8.663 \times 10^{-2}$ & $8.666 \times 10^{-4}$ & $8.667 \times 10^{-6}$ & $1.722 \times 10^{-1}$ & $1.721 \times 10^{-3}$ & $1.721 \times 10^{-5}$ & $4.285 \times 10^{-1}$ & $4.306 \times 10^{-3}$ & $4.307 \times 10^{-5}$ \\
\hline midpointqp & $8.673 \times 10^{-2}$ & $8.669 \times 10^{-4}$ & $8.669 \times 10^{-6}$ & $1.723 \times 10^{-1}$ & $1.721 \times 10^{-3}$ & $1.721 \times 10^{-5}$ & $4.289 \times 10^{-1}$ & $4.307 \times 10^{-3}$ & $4.307 \times 10^{-5}$ \\
\hline PureS/Sol. & & $\mathrm{T}=1000$ & & & $T=2000$ & & & $T=5000$ & \\
\hline $\mathrm{dT}$ & 0.01 & 0.001 & 0.0001 & 0.01 & 0.001 & 0.0001 & 0.01 & 0.001 & 0.0001 \\
\hline sv2symp & $3.707 \times 10^{-4}$ & $3.706 \times 10^{-6}$ & $3.706 \times 10^{-8}$ & $7.05 \times 10^{-4}$ & $7.05 \times 10^{-6}$ & $7.05 \times 10^{-8}$ & $1.712 \times 10^{-3}$ & $1.712 \times 10^{-5}$ & $1.712 \times 10^{-7}$ \\
\hline midpoint & $1.485 \times 10^{-3}$ & $1.447 \times 10^{-5}$ & $1.442 \times 10^{-7}$ & $2.917 \times 10^{-3}$ & $2.756 \times 10^{-5}$ & $2.742 \times 10^{-7}$ & $7.791 \times 10^{-3}$ & $6.756 \times 10^{-5}$ & $6.64 \times 10^{-7}$ \\
\hline midpointqp & $1.507 \times 10^{-3}$ & $1.467 \times 10^{-5}$ & $1.463 \times 10^{-7}$ & $2.964 \times 10^{-3}$ & $2.798 \times 10^{-5}$ & $2.781 \times 10^{-7}$ & $7.932 \times 10^{-3}$ & $6.865 \times 10^{-5}$ & $6.758 \times 10^{-7}$ \\
\hline PureS/Disp. & & $\mathrm{T}=1000$ & & & $\mathrm{~T}=2000$ & & & $T=5000$ & \\
\hline $\mathrm{dT}$ & 0.01 & 0.001 & 0.0001 & 0.01 & 0.001 & 0.0001 & 0.01 & 0.001 & 0.0001 \\
\hline sv2symp & $1.295 \times 10^{-5}$ & $1.295 \times 10^{-5}$ & $1.295 \times 10^{-5}$ & $6.467 \times 10^{-6}$ & $6.467 \times 10^{-6}$ & $6.467 \times 10^{-6}$ & $2.586 \times 10^{-6}$ & $2.586 \times 10^{-6}$ & $2.586 \times 10^{-6}$ \\
\hline midpoint & $1.295 \times 10^{-5}$ & $1.295 \times 10^{-5}$ & $1.295 \times 10^{-5}$ & $6.469 \times 10^{-6}$ & $6.467 \times 10^{-6}$ & $6.467 \times 10^{-6}$ & $2.588 \times 10^{-6}$ & $2.586 \times 10^{-6}$ & $2.586 \times 10^{-6}$ \\
\hline midpointqp & $1.295 \times 10^{-5}$ & $1.295 \times 10^{-5}$ & $1.295 \times 10^{-5}$ & $6.469 \times 10^{-6}$ & $6.467 \times 10^{-6}$ & $6.467 \times 10^{-6}$ & $2.588 \times 10^{-6}$ & $2.586 \times 10^{-6}$ & $2.586 \times 10^{-6}$ \\
\hline
\end{tabular}

B.2. Second order - errors for $b_{n}(t)$.

\begin{tabular}{|c|c|c|c|c|c|c|c|c|c|}
\hline quad/Sol. & & $\mathrm{T}=1000$ & & & $\mathrm{~T}=2000$ & & & $\mathrm{~T}=5000$ & \\
\hline $\mathrm{dT}$ & 0.01 & 0.001 & 0.0001 & 0.01 & 0.001 & 0.0001 & 0.01 & 0.001 & 0.0001 \\
\hline sv2symp & $4.471 \times 10^{-3}$ & $4.47 \times 10^{-5}$ & $4.47 \times 10^{-7}$ & $8.82 \times 10^{-3}$ & $8.819 \times 10^{-5}$ & $8.819 \times 10^{-7}$ & $2.197 \times 10^{-2}$ & $2.196 \times 10^{-4}$ & $2.196 \times 10^{-6}$ \\
\hline midpoint & $1.823 \times 10^{-2}$ & $1.432 \times 10^{-4}$ & $1.393 \times 10^{-6}$ & $4.176 \times 10^{-2}$ & $2.882 \times 10^{-4}$ & $2.753 \times 10^{-6}$ & $1.471 \times 10^{-1}$ & $7.608 \times 10^{-4}$ & $6.898 \times 10^{-6}$ \\
\hline midpointqp & $1.992 \times 10^{-2}$ & $1.551 \times 10^{-4}$ & $1.507 \times 10^{-6}$ & $4.558 \times 10^{-2}$ & $3.12 \times 10^{-4}$ & $2.976 \times 10^{-6}$ & $1.603 \times 10^{-1}$ & $8.234 \times 10^{-4}$ & $7.456 \times 10^{-6}$ \\
\hline
\end{tabular}

\begin{tabular}{|c|c|c|c|c|c|c|c|c|c|}
\hline quad/Disp. & & $\mathrm{T}=1000$ & & & $\mathrm{~T}=2000$ & & \\
\hline $\mathrm{dT}$ & 0.01 & 0.001 & 0.0001 & 0.01 & 0.001 & 0.0001 & 0.01 & 0.001 & 0.0001 \\
\hline sv2symp & $2.18 \times 10^{-2}$ & $2.18 \times 10^{-4}$ & $2.181 \times 10^{-6}$ & $4.394 \times 10^{-2}$ & $4.392 \times 10^{-4}$ & $4.392 \times 10^{-6}$ & $1.082 \times 10^{-1}$ & $1.082 \times 10^{-3}$ & $1.082 \times 10^{-5}$ \\
\hline midpoint & $8.761 \times 10^{-2}$ & $8.745 \times 10^{-4}$ & $8.745 \times 10^{-6}$ & $1.768 \times 10^{-1}$ & $1.761 \times 10^{-3}$ & $1.761 \times 10^{-5}$ & $4.327 \times 10^{-1}$ & $4.337 \times 10^{-3}$ & $4.328 \times 10^{-5}$ \\
\hline midpointqp & $8.798 \times 10^{-2}$ & $8.749 \times 10^{-4}$ & $8.746 \times 10^{-6}$ & $1.775 \times 10^{-1}$ & $1.762 \times 10^{-3}$ & $1.761 \times 10^{-5}$ & $4.345 \times 10^{-1}$ & $4.338 \times 10^{-3}$ & $4.335 \times 10^{-5}$ \\
\hline
\end{tabular}

\begin{tabular}{|c|c|c|c|c|c|c|c|c|c|}
\hline dirac/Sol. & & $\mathrm{T}=1000$ & & & $\mathrm{~T}=2000$ & & \\
\hline $\mathrm{dT}$ & 0.01 & 0.001 & 0.0001 & 0.01 & 0.001 & 0.0001 & 0.01 & 0.001 \\
\hline sv2symp & $1.257 \times 10^{-1}$ & $1.046 \times 10^{-3}$ & $1.045 \times 10^{-5}$ & $3.659 \times 10^{-1}$ & $4.826 \times 10^{-3}$ & $4.835 \times 10^{-5}$ & $9.797 \times 10^{-1}$ & $1.175 \times 10^{-2}$ & $1.169 \times 10^{-4}$ \\
\hline midpoint & 1.356 & $1.015 \times 10^{-2}$ & $3.231 \times 10^{-5}$ & 1.445 & $7.853 \times 10^{-2}$ & $1.846 \times 10^{-4}$ & 1.567 & $4.909 \times 10^{-1}$ & $6.994 \times 10^{-4}$ \\
\hline midpointqp & 1.358 & $1.365 \times 10^{-2}$ & $4.567 \times 10^{-5}$ & 1.546 & $1.026 \times 10^{-1}$ & $2.569 \times 10^{-4}$ & 1.638 & $6.32 \times 10^{-1}$ & $9.508 \times 10^{-4}$ \\
\hline
\end{tabular}




\begin{tabular}{|c|c|c|c|c|c|c|c|c|c|}
\hline dirac/Disp. & & $\mathrm{T}=1000$ & & & $\mathrm{~T}=2000$ & & & $\mathrm{~T}=5000$ & \\
\hline$\overline{d T}$ & 0.01 & 0.001 & 0.0001 & 0.01 & 0.001 & 0.0001 & 0.01 & 0.001 & 0.0001 \\
\hline sv2symp & $2.17 \times 10^{-2}$ & $2.17 \times 10^{-4}$ & $2.17 \times 10^{-6}$ & $4.377 \times 10^{-2}$ & $4.377 \times 10^{-4}$ & $4.377 \times 10^{-6}$ & $1.073 \times 10^{-1}$ & $1.074 \times 10^{-3}$ & $1.074 \times 10^{-5}$ \\
\hline midpoint & $1.018 \times 10^{-1}$ & $9.005 \times 10^{-4}$ & $8.892 \times 10^{-6}$ & $2.041 \times 10^{-1}$ & $1.799 \times 10^{-3}$ & $1.776 \times 10^{-5}$ & $5.004 \times 10^{-1}$ & $4.383 \times 10^{-3}$ & $4.326 \times 10^{-5}$ \\
\hline midpointqp & $1.109 \times 10^{-1}$ & $8.992 \times 10^{-4}$ & $8.79 \times 10^{-6}$ & $2.244 \times 10^{-1}$ & $1.806 \times 10^{-3}$ & $1.765 \times 10^{-5}$ & $5.568 \times 10^{-1}$ & $4.42 \times 10^{-3}$ & $4.318 \times 10^{-5}$ \\
\hline
\end{tabular}

\begin{tabular}{|c|c|c|c|c|c|c|c|c|c|}
\hline double/Sol. & & $\mathrm{T}=1000$ & & & $T=2000$ & & & $\mathrm{~T}=5000$ & \\
\hline $\mathrm{dT}$ & 0.01 & 0.001 & 0.0001 & 0.01 & 0.001 & 0.0001 & 0.01 & 0.001 & 0.0001 \\
\hline sv2symp & $1.723 \times 10^{-3}$ & $1.723 \times 10^{-5}$ & $1.723 \times 10^{-7}$ & $3.387 \times 10^{-3}$ & $3.386 \times 10^{-5}$ & $3.386 \times 10^{-7}$ & $8.373 \times 10^{-3}$ & $8.372 \times 10^{-5}$ & $8.372 \times 10^{-7}$ \\
\hline midpoint & $7.758 \times 10^{-3}$ & $7.096 \times 10^{-5}$ & $7.029 \times 10^{-7}$ & $1.588 \times 10^{-2}$ & $1.403 \times 10^{-4}$ & $1.385 \times 10^{-6}$ & $4.363 \times 10^{-2}$ & $3.518 \times 10^{-4}$ & $3.432 \times 10^{-6}$ \\
\hline midpointqp & $8.848 \times 10^{-3}$ & $8.086 \times 10^{-5}$ & $8.011 \times 10^{-7}$ & $1.804 \times 10^{-2}$ & $1.597 \times 10^{-4}$ & $1.577 \times 10^{-6}$ & $4.916 \times 10^{-2}$ & $3.996 \times 10^{-4}$ & $3.905 \times 10^{-6}$ \\
\hline
\end{tabular}

\begin{tabular}{|c|c|c|c|c|c|c|c|c|c|}
\hline double/Disp. & & $\mathrm{T}=1000$ & & & $T=2000$ & & & $T=5000$ & \\
\hline $\mathrm{dT}$ & 0.01 & 0.001 & 0.0001 & 0.01 & 0.001 & 0.0001 & 0.01 & 0.001 & 0.0001 \\
\hline sv2symp & $2.125 \times 10^{-2}$ & $2.125 \times 10^{-4}$ & $2.125 \times 10^{-6}$ & $4.355 \times 10^{-2}$ & $4.355 \times 10^{-4}$ & $4.355 \times 10^{-6}$ & $1.074 \times 10^{-1}$ & $1.074 \times 10^{-3}$ & $1.074 \times 10^{-5}$ \\
\hline midpoint & $8.478 \times 10^{-2}$ & $8.49 \times 10^{-4}$ & $8.492 \times 10^{-6}$ & $1.737 \times 10^{-1}$ & $1.741 \times 10^{-3}$ & $1.741 \times 10^{-5}$ & $4.267 \times 10^{-1}$ & $4.294 \times 10^{-3}$ & $4.293 \times 10^{-5}$ \\
\hline midpointqp & $8.497 \times 10^{-2}$ & $8.492 \times 10^{-4}$ & $8.492 \times 10^{-6}$ & $1.741 \times 10^{-1}$ & $1.741 \times 10^{-3}$ & $1.741 \times 10^{-5}$ & $4.277 \times 10^{-1}$ & $4.295 \times 10^{-3}$ & $4.295 \times 10^{-5}$ \\
\hline
\end{tabular}

\begin{tabular}{|c|c|c|c|c|c|c|c|c|c|}
\hline NoS/Sol. & & $\mathrm{T}=1000$ & & & $T=2000$ & & & $T=5000$ & \\
\hline $\mathrm{dT}$ & 0.01 & 0.001 & 0.0001 & 0.01 & 0.001 & 0.0001 & 0.01 & 0.001 & 0.0001 \\
\hline sv2symp & $1.849 \times 10^{-5}$ & $1.849 \times 10^{-7}$ & $1.849 \times 10^{-9}$ & $2.528 \times 10^{-5}$ & $2.528 \times 10^{-7}$ & $2.529 \times 10^{-9}$ & $3.275 \times 10^{-5}$ & $3.275 \times 10^{-7}$ & $3.276 \times 10^{-9}$ \\
\hline midpoint & $7.965 \times 10^{-5}$ & $7.925 \times 10^{-7}$ & $7.981 \times 10^{-9}$ & $1.076 \times 10^{-4}$ & $1.071 \times 10^{-6}$ & $1.079 \times 10^{-8}$ & $1.373 \times 10^{-4}$ & $1.369 \times 10^{-6}$ & $1.461 \times 10^{-8}$ \\
\hline midpointqp & $8.245 \times 10^{-5}$ & $8.193 \times 10^{-7}$ & $8.188 \times 10^{-9}$ & $1.106 \times 10^{-4}$ & $1.1 \times 10^{-6}$ & $1.099 \times 10^{-8}$ & $1.4 \times 10^{-4}$ & $1.394 \times 10^{-6}$ & $1.394 \times 10^{-8}$ \\
\hline
\end{tabular}

\begin{tabular}{|c|c|c|c|c|c|c|c|c|c|}
\hline NoS/Disp. & & $\mathrm{T}=1000$ & & & $\mathrm{~T}=2000$ & & $\mathrm{~T}=5000$ \\
\hline $\mathrm{dT}$ & 0.01 & 0.001 & 0.0001 & 0.01 & 0.001 & 0.0001 & 0.01 & 0.001 & 0.0001 \\
\hline sv2symp & $2.143 \times 10^{-2}$ & $2.143 \times 10^{-4}$ & $2.143 \times 10^{-6}$ & $4.282 \times 10^{-2}$ & $4.282 \times 10^{-4}$ & $4.282 \times 10^{-6}$ & $1.089 \times 10^{-1}$ & $1.089 \times 10^{-3}$ & $1.089 \times 10^{-5}$ \\
\hline midpoint & $8.568 \times 10^{-2}$ & $8.569 \times 10^{-4}$ & $8.57 \times 10^{-6}$ & $1.711 \times 10^{-1}$ & $1.713 \times 10^{-3}$ & $1.713 \times 10^{-5}$ & $4.328 \times 10^{-1}$ & $4.354 \times 10^{-3}$ & $4.354 \times 10^{-5}$ \\
\hline midpointqp & $8.578 \times 10^{-2}$ & $8.572 \times 10^{-4}$ & $8.572 \times 10^{-6}$ & $1.712 \times 10^{-1}$ & $1.713 \times 10^{-3}$ & $1.713 \times 10^{-5}$ & $4.332 \times 10^{-1}$ & $4.354 \times 10^{-3}$ & $4.354 \times 10^{-5}$ \\
\hline
\end{tabular}

\begin{tabular}{|c|c|c|c|c|c|c|c|c|c|}
\hline PureS/Sol. & & $\mathrm{T}=1000$ & & & $T=2000$ & & & $\mathrm{~T}=5000$ & \\
\hline $\mathrm{dT}$ & 0.01 & 0.001 & 0.0001 & 0.01 & 0.001 & 0.0001 & 0.01 & 0.001 & 0.0001 \\
\hline sv2symp & $3.703 \times 10^{-4}$ & $3.703 \times 10^{-6}$ & $3.702 \times 10^{-8}$ & $7.047 \times 10^{-4}$ & $7.047 \times 10^{-6}$ & $7.047 \times 10^{-8}$ & $1.712 \times 10^{-3}$ & $1.712 \times 10^{-5}$ & $1.712 \times 10^{-7}$ \\
\hline midpoint & $1.485 \times 10^{-3}$ & $1.447 \times 10^{-5}$ & $1.442 \times 10^{-7}$ & $2.916 \times 10^{-3}$ & $2.755 \times 10^{-5}$ & $2.742 \times 10^{-7}$ & $7.789 \times 10^{-3}$ & $6.755 \times 10^{-5}$ & $6.639 \times 10^{-7}$ \\
\hline midpointqp & $1.506 \times 10^{-3}$ & $1.467 \times 10^{-5}$ & $1.463 \times 10^{-7}$ & $2.963 \times 10^{-3}$ & $2.797 \times 10^{-5}$ & $2.781 \times 10^{-7}$ & $7.93 \times 10^{-3}$ & $6.864 \times 10^{-5}$ & $6.757 \times 10^{-7}$ \\
\hline
\end{tabular}

\begin{tabular}{|c|c|c|c|c|c|c|c|c|c|}
\hline PureS/Disp. & & $\mathrm{T}=1000$ & & & $\mathrm{~T}=2000$ & & \\
\hline $\mathrm{dT}$ & 0.01 & 0.001 & 0.0001 & 0.01 & 0.001 & 0.0001 & 0.01 & 0.001 & 0.0001 \\
\hline sv2symp & $2.589 \times 10^{-5}$ & $2.589 \times 10^{-5}$ & $2.589 \times 10^{-5}$ & $1.293 \times 10^{-5}$ & $1.293 \times 10^{-5}$ & $1.293 \times 10^{-5}$ & $5.173 \times 10^{-6}$ & $5.172 \times 10^{-6}$ & $5.172 \times 10^{-6}$ \\
\hline midpoint & $2.59 \times 10^{-5}$ & $2.589 \times 10^{-5}$ & $2.589 \times 10^{-5}$ & $1.294 \times 10^{-5}$ & $1.293 \times 10^{-5}$ & $1.293 \times 10^{-5}$ & $5.175 \times 10^{-6}$ & $5.172 \times 10^{-6}$ & $5.172 \times 10^{-6}$ \\
\hline midpointqp & $2.59 \times 10^{-5}$ & $2.589 \times 10^{-5}$ & $2.589 \times 10^{-5}$ & $1.294 \times 10^{-5}$ & $1.293 \times 10^{-5}$ & $1.293 \times 10^{-5}$ & $5.175 \times 10^{-6}$ & $5.172 \times 10^{-6}$ & $5.172 \times 10^{-6}$ \\
\hline
\end{tabular}


B.3. Fourth order - errors for $a_{n}(t)$.

\begin{tabular}{|c|c|c|c|c|c|c|c|c|c|}
\hline quad/Sol. & & $\mathrm{T}=1000$ & & & $T=2000$ & & & $T=5000$ & \\
\hline $\mathrm{dT}$ & 0.01 & 0.001 & 0.0001 & 0.01 & 0.001 & 0.0001 & 0.01 & 0.001 & 0.0001 \\
\hline rk4 & $2.865 \times 10^{-7}$ & $5.51 \times 10^{-10}$ & $5.898 \times 10^{-10}$ & $6.509 \times 10^{-7}$ & $1.476 \times 10^{-10}$ & $2.968 \times 10^{-9}$ & $2.228 \times 10^{-6}$ & $9.889 \times 10^{-10}$ & $2.031 \times 10^{-8}$ \\
\hline rk4qp & $3.27 \times 10^{-7}$ & $5.507 \times 10^{-10}$ & $5.501 \times 10^{-10}$ & $7.39 \times 10^{-7}$ & $5.353 \times 10^{-11}$ & $2.099 \times 10^{-11}$ & $2.511 \times 10^{-6}$ & $1.453 \times 10^{-10}$ & $6.118 \times 10^{-11}$ \\
\hline rkf45 & $6.78 \times 10^{-8}$ & $5.502 \times 10^{-10}$ & $5.904 \times 10^{-10}$ & $1.685 \times 10^{-7}$ & $1.296 \times 10^{-10}$ & $2.972 \times 10^{-9}$ & $6.68 \times 10^{-7}$ & $9.647 \times 10^{-10}$ & $2.032 \times 10^{-8}$ \\
\hline$a b 4$ & $3.533 \times 10^{-3}$ & $3.53 \times 10^{-5}$ & $3.527 \times 10^{-7}$ & $7.064 \times 10^{-3}$ & $7.063 \times 10^{-5}$ & $7.055 \times 10^{-7}$ & $1.745 \times 10^{-2}$ & $1.751 \times 10^{-4}$ & $1.749 \times 10^{-6}$ \\
\hline
\end{tabular}

\begin{tabular}{|c|c|c|c|c|c|c|c|c|c|}
\hline quad/Disp. & & $\mathrm{T}=1000$ & & & $\mathrm{~T}=2000$ & & & $\mathrm{~T}=5000$ & \\
\hline $\mathrm{dT}$ & 0.01 & 0.001 & 0.0001 & 0.01 & 0.001 & 0.0001 & 0.01 & 0.001 & 0.0001 \\
\hline rk4 & $1.371 \times 10^{-6}$ & $3.443 \times 10^{-9}$ & $3.466 \times 10^{-9}$ & $2.732 \times 10^{-6}$ & $7.455 \times 10^{-9}$ & $8.864 \times 10^{-9}$ & $6.599 \times 10^{-6}$ & $1.172 \times 10^{-8}$ & $7.7 \times 10^{-8}$ \\
\hline rk4qp & $1.381 \times 10^{-6}$ & $3.444 \times 10^{-9}$ & $3.468 \times 10^{-9}$ & $2.75 \times 10^{-6}$ & $7.424 \times 10^{-9}$ & $7.356 \times 10^{-9}$ & $6.638 \times 10^{-6}$ & $1.12 \times 10^{-8}$ & $1.13 \times 10^{-8}$ \\
\hline rkf45 & $2.129 \times 10^{-7}$ & $3.471 \times 10^{-9}$ & $3.466 \times 10^{-9}$ & $4.216 \times 10^{-7}$ & $7.379 \times 10^{-9}$ & $8.864 \times 10^{-9}$ & $1.023 \times 10^{-6}$ & $1.152 \times 10^{-8}$ & $7.685 \times 10^{-8}$ \\
\hline$a b 4$ & $4.195 \times 10^{-4}$ & $3.768 \times 10^{-6}$ & $3.747 \times 10^{-8}$ & $5.5 \times 10^{-4}$ & $4.546 \times 10^{-6}$ & $4.976 \times 10^{-8}$ & $7.508 \times 10^{-4}$ & $4.96 \times 10^{-6}$ & $1.228 \times 10^{-7}$ \\
\hline
\end{tabular}

\begin{tabular}{|c|c|c|c|c|c|c|c|c|c|}
\hline dirac/Sol. & & $\mathrm{T}=1000$ & & & $T=2000$ & & & $\mathrm{~T}=5000$ & \\
\hline $\mathrm{dT}$ & 0.01 & 0.001 & 0.0001 & 0.01 & 0.001 & 0.0001 & 0.01 & 0.001 & 0.0001 \\
\hline rk4 & $3.284 \times 10^{-3}$ & $4.11 \times 10^{-8}$ & $3.874 \times 10^{-11}$ & $5.916 \times 10^{-3}$ & $6.747 \times 10^{-8}$ & $1.03 \times 10^{-10}$ & $4.282 \times 10^{-2}$ & $4.228 \times 10^{-7}$ & $6.462 \times 10^{-10}$ \\
\hline rk4qp & $4.241 \times 10^{-3}$ & $5.391 \times 10^{-8}$ & $2.022 \times 10^{-11}$ & $7.602 \times 10^{-3}$ & $8.783 \times 10^{-8}$ & $7.222 \times 10^{-11}$ & $5.61 \times 10^{-2}$ & $5.468 \times 10^{-7}$ & $6.104 \times 10^{-11}$ \\
\hline rkf 45 & $1.621 \times 10^{-3}$ & $1.759 \times 10^{-8}$ & $7.666 \times 10^{-11}$ & 3. $\times 10^{-3}$ & $3.113 \times 10^{-8}$ & $6.779 \times 10^{-11}$ & $1.947 \times 10^{-2}$ & $2.051 \times 10^{-7}$ & $3.397 \times 10^{-10}$ \\
\hline$a b 4$ & $2.952 \times 10^{-1}$ & $1.09 \times 10^{-3}$ & $1.095 \times 10^{-5}$ & $6.553 \times 10^{-1}$ & $1.01 \times 10^{-3}$ & $1.016 \times 10^{-5}$ & 1.28 & $2.692 \times 10^{-3}$ & $2.755 \times 10^{-5}$ \\
\hline
\end{tabular}

\begin{tabular}{|c|c|c|c|c|c|c|c|c|c|}
\hline dirac/Disp. & & $\mathrm{T}=1000$ & & & $\mathrm{~T}=2000$ & & & $T=5000$ & \\
\hline $\mathrm{dT}$ & 0.01 & 0.001 & 0.0001 & 0.01 & 0.001 & 0.0001 & 0.01 & 0.001 & 0.0001 \\
\hline rk4 & $3.676 \times 10^{-6}$ & $1.685 \times 10^{-10}$ & $2.575 \times 10^{-10}$ & $7.051 \times 10^{-6}$ & $3.221 \times 10^{-10}$ & $8.182 \times 10^{-10}$ & $1.722 \times 10^{-5}$ & $1.122 \times 10^{-9}$ & $6.378 \times 10^{-9}$ \\
\hline rk4qp & $5.037 \times 10^{-6}$ & $1.86 \times 10^{-10}$ & $6.011 \times 10^{-11}$ & $9.665 \times 10^{-6}$ & $3.503 \times 10^{-10}$ & $8.565 \times 10^{-11}$ & $2.362 \times 10^{-5}$ & $8.024 \times 10^{-10}$ & $1.398 \times 10^{-10}$ \\
\hline rkf 45 & $1.503 \times 10^{-6}$ & $3.749 \times 10^{-11}$ & $2.449 \times 10^{-10}$ & $2.94 \times 10^{-6}$ & $3.258 \times 10^{-11}$ & $9.732 \times 10^{-10}$ & $7.283 \times 10^{-6}$ & $3.389 \times 10^{-10}$ & $6.417 \times 10^{-9}$ \\
\hline$a b 4$ & $5.565 \times 10^{-3}$ & $5.217 \times 10^{-5}$ & $5.223 \times 10^{-7}$ & $6.307 \times 10^{-3}$ & $5.578 \times 10^{-5}$ & $5.59 \times 10^{-7}$ & $8.02 \times 10^{-3}$ & $6.146 \times 10^{-5}$ & $6.204 \times 10^{-7}$ \\
\hline
\end{tabular}

\begin{tabular}{|c|c|c|c|c|c|c|c|c|c|}
\hline double/Sol. & & $T=1000$ & & & $T=2000$ & & & $\mathrm{~T}=5000$ & \\
\hline $\mathrm{dT}$ & 0.01 & 0.001 & 0.0001 & 0.01 & 0.001 & 0.0001 & 0.01 & 0.001 & 0.0001 \\
\hline rk4 & $1.001 \times 10^{-7}$ & $2.442 \times 10^{-11}$ & $1.786 \times 10^{-10}$ & $2.006 \times 10^{-7}$ & $6.527 \times 10^{-11}$ & $5.316 \times 10^{-10}$ & $5.291 \times 10^{-7}$ & $4.041 \times 10^{-10}$ & $1.666 \times 10^{-8}$ \\
\hline$r k 4 q p$ & $1.129 \times 10^{-7}$ & $9.596 \times 10^{-12}$ & $2.287 \times 10^{-12}$ & $2.256 \times 10^{-7}$ & $1.868 \times 10^{-11}$ & $6.062 \times 10^{-12}$ & $5.923 \times 10^{-7}$ & $4.655 \times 10^{-11}$ & $7.707 \times 10^{-11}$ \\
\hline rkf45 & $1.867 \times 10^{-8}$ & $1.009 \times 10^{-11}$ & $1.788 \times 10^{-10}$ & $3.843 \times 10^{-8}$ & $3.121 \times 10^{-11}$ & $5.324 \times 10^{-10}$ & $1.083 \times 10^{-7}$ & $3.049 \times 10^{-10}$ & $1.666 \times 10^{-8}$ \\
\hline$a b 4$ & $4.271 \times 10^{-3}$ & $4.284 \times 10^{-5}$ & $4.284 \times 10^{-7}$ & $8.436 \times 10^{-3}$ & $8.463 \times 10^{-5}$ & $8.461 \times 10^{-7}$ & $2.093 \times 10^{-2}$ & $2.1 \times 10^{-4}$ & $2.099 \times 10^{-6}$ \\
\hline
\end{tabular}

\begin{tabular}{|c|c|c|c|c|c|c|c|c|c|}
\hline double/Disp. & & $\mathrm{T}=1000$ & & & $T=2000$ & & & $\mathrm{~T}=5000$ & \\
\hline $\mathrm{dT}$ & 0.01 & 0.001 & 0.0001 & 0.01 & 0.001 & 0.0001 & 0.01 & 0.001 & 0.0001 \\
\hline rk4 & $1.317 \times 10^{-6}$ & $1.381 \times 10^{-10}$ & $7.958 \times 10^{-11}$ & $2.595 \times 10^{-6}$ & $2.79 \times 10^{-10}$ & $2.274 \times 10^{-10}$ & $6.58 \times 10^{-6}$ & $1 . \times 10^{-9}$ & $1.58 \times 10^{-8}$ \\
\hline rk4qp & $1.32 \times 10^{-6}$ & $1.312 \times 10^{-10}$ & $1.007 \times 10^{-11}$ & $2.602 \times 10^{-6}$ & $2.608 \times 10^{-10}$ & $1.522 \times 10^{-11}$ & $6.598 \times 10^{-6}$ & $6.569 \times 10^{-10}$ & $5.339 \times 10^{-11}$ \\
\hline rkf 45 & $2.02 \times 10^{-7}$ & $1.81 \times 10^{-11}$ & $8.081 \times 10^{-11}$ & $3.981 \times 10^{-7}$ & $2.337 \times 10^{-11}$ & $2.324 \times 10^{-10}$ & $1.009 \times 10^{-6}$ & $2.204 \times 10^{-10}$ & $1.581 \times 10^{-8}$ \\
\hline$a b 4$ & $3.715 \times 10^{-4}$ & $3.203 \times 10^{-6}$ & $3.206 \times 10^{-8}$ & $4.441 \times 10^{-4}$ & $3.416 \times 10^{-6}$ & $3.429 \times 10^{-8}$ & $6.49 \times 10^{-4}$ & $3.844 \times 10^{-6}$ & $5.351 \times 10^{-8}$ \\
\hline
\end{tabular}




\begin{tabular}{|c|c|c|c|c|c|c|c|c|c|}
\hline NoS/Sol. & & $\mathrm{T}=1000$ & & & $\mathrm{~T}=2000$ & & $\mathrm{~T}=5000$ & & \\
\hline $\mathrm{dT}$ & 0.01 & 0.001 & 0.0001 & 0.01 & 0.001 & 0.0001 & 0.01 & 0.001 & 0.0001 \\
\hline $\mathrm{rk4}$ & $2.405 \times 10^{-10}$ & $8.695 \times 10^{-11}$ & $8.286 \times 10^{-10}$ & $1.726 \times 10^{-10}$ & $1.418 \times 10^{-10}$ & $1.287 \times 10^{-9}$ & $1.282 \times 10^{-10}$ & $4.785 \times 10^{-10}$ & $4.865 \times 10^{-9}$ \\
\hline $\mathrm{rk4qp}$ & $2.577 \times 10^{-10}$ & $3.684 \times 10^{-11}$ & $3.684 \times 10^{-11}$ & $1.815 \times 10^{-10}$ & $1.949 \times 10^{-11}$ & $1.949 \times 10^{-11}$ & $1.214 \times 10^{-10}$ & $9.323 \times 10^{-12}$ & $9.33 \times 10^{-12}$ \\
\hline $\mathrm{rkf} 45$ & $5.713 \times 10^{-11}$ & $8.667 \times 10^{-11}$ & $8.286 \times 10^{-10}$ & $4.106 \times 10^{-11}$ & $1.415 \times 10^{-10}$ & $1.287 \times 10^{-9}$ & $5.762 \times 10^{-11}$ & $4.79 \times 10^{-10}$ & $4.865 \times 10^{-9}$ \\
\hline $\mathrm{ab} 4$ & $1.217 \times 10^{-5}$ & $1.211 \times 10^{-7}$ & $1.46 \times 10^{-9}$ & $1.149 \times 10^{-5}$ & $1.143 \times 10^{-7}$ & $1.727 \times 10^{-9}$ & $1 . \times 10^{-5}$ & $9.939 \times 10^{-8}$ & $4.972 \times 10^{-9}$ \\
\hline
\end{tabular}

\begin{tabular}{|c|c|c|c|c|c|c|c|c|c|}
\hline NoS/Disp. & & $\mathrm{T}=1000$ & & & $T=2000$ & & & $\mathrm{~T}=5000$ & \\
\hline $\mathrm{dT}$ & 0.01 & 0.001 & 0.0001 & 0.01 & 0.001 & 0.0001 & 0.01 & 0.001 & 0.0001 \\
\hline rk4 & $1.299 \times 10^{-6}$ & $1.335 \times 10^{-10}$ & $4.173 \times 10^{-11}$ & $2.578 \times 10^{-6}$ & $2.697 \times 10^{-10}$ & $5.083 \times 10^{-11}$ & $6.454 \times 10^{-6}$ & $6.209 \times 10^{-10}$ & $2.603 \times 10^{-10}$ \\
\hline rk4qp & $1.3 \times 10^{-6}$ & $1.294 \times 10^{-10}$ & $1.311 \times 10^{-11}$ & $2.582 \times 10^{-6}$ & $2.607 \times 10^{-10}$ & $1.954 \times 10^{-11}$ & $6.462 \times 10^{-6}$ & $6.337 \times 10^{-10}$ & $4.847 \times 10^{-11}$ \\
\hline rkf45 & $1.997 \times 10^{-7}$ & $2.053 \times 10^{-11}$ & $4.3 \times 10^{-11}$ & $3.963 \times 10^{-7}$ & $3.68 \times 10^{-11}$ & $5.154 \times 10^{-11}$ & $9.92 \times 10^{-7}$ & $1.46 \times 10^{-10}$ & $2.212 \times 10^{-10}$ \\
\hline$a b 4$ & $9.795 \times 10^{-5}$ & $6.17 \times 10^{-7}$ & $6.166 \times 10^{-9}$ & $1.503 \times 10^{-4}$ & $6.476 \times 10^{-7}$ & $6.439 \times 10^{-9}$ & $3.12 \times 10^{-4}$ & $6.84 \times 10^{-7}$ & $6.546 \times 10^{-9}$ \\
\hline
\end{tabular}

\begin{tabular}{|c|c|c|c|c|c|c|c|c|c|}
\hline PureS/Sol. & & $\mathrm{T}=1000$ & & & $T=2000$ & & & $\mathrm{~T}=5000$ & \\
\hline $\mathrm{dT}$ & 0.01 & 0.001 & 0.0001 & 0.01 & 0.001 & 0.0001 & 0.01 & 0.001 & 0.0001 \\
\hline rk4 & $4.753 \times 10^{-9}$ & $1.185 \times 10^{-10}$ & $2.224 \times 10^{-10}$ & $9.438 \times 10^{-9}$ & $2.965 \times 10^{-10}$ & $2.793 \times 10^{-10}$ & $2.631 \times 10^{-8}$ & $2.683 \times 10^{-10}$ & $1.294 \times 10^{-9}$ \\
\hline rk4qp & $4.881 \times 10^{-9}$ & $2.739 \times 10^{-11}$ & $2.744 \times 10^{-11}$ & $9.722 \times 10^{-9}$ & $1.063 \times 10^{-11}$ & $1.104 \times 10^{-11}$ & $2.714 \times 10^{-8}$ & $1.619 \times 10^{-12}$ & $3.488 \times 10^{-12}$ \\
\hline rkf45 & $7.944 \times 10^{-10}$ & $3.156 \times 10^{-11}$ & $8.664 \times 10^{-10}$ & $1.693 \times 10^{-9}$ & $3.015 \times 10^{-11}$ & $2.611 \times 10^{-9}$ & $5.422 \times 10^{-9}$ & $1.172 \times 10^{-10}$ & $1.265 \times 10^{-9}$ \\
\hline$a b 4$ & $7.725 \times 10^{-5}$ & $7.743 \times 10^{-7}$ & $7.677 \times 10^{-9}$ & $1.579 \times 10^{-4}$ & $1.583 \times 10^{-6}$ & $1.592 \times 10^{-8}$ & $4.003 \times 10^{-4}$ & $4.016 \times 10^{-6}$ & $3.879 \times 10^{-8}$ \\
\hline
\end{tabular}

\begin{tabular}{|c|c|c|c|c|c|c|c|c|c|}
\hline PureS/Disp. & & $\mathrm{T}=1000$ & & & $\mathrm{~T}=2000$ & & \\
\hline $\mathrm{dT}$ & 0.01 & 0.001 & 0.0001 & 0.01 & 0.001 & 0.0001 & 0.01 & 0.001 & 0.0001 \\
\hline $\mathrm{rk} 4$ & $1.295 \times 10^{-5}$ & $1.295 \times 10^{-5}$ & $1.295 \times 10^{-5}$ & $6.467 \times 10^{-6}$ & $6.467 \times 10^{-6}$ & $6.467 \times 10^{-6}$ & $2.586 \times 10^{-6}$ & $2.586 \times 10^{-6}$ & $2.586 \times 10^{-6}$ \\
\hline $\mathrm{rk4qp}$ & $1.295 \times 10^{-5}$ & $1.295 \times 10^{-5}$ & $1.295 \times 10^{-5}$ & $6.467 \times 10^{-6}$ & $6.467 \times 10^{-6}$ & $6.467 \times 10^{-6}$ & $2.586 \times 10^{-6}$ & $2.586 \times 10^{-6}$ & $2.586 \times 10^{-6}$ \\
\hline $\mathrm{rkf} 45$ & $1.295 \times 10^{-5}$ & $1.295 \times 10^{-5}$ & $1.295 \times 10^{-5}$ & $6.467 \times 10^{-6}$ & $6.467 \times 10^{-6}$ & $6.467 \times 10^{-6}$ & $2.586 \times 10^{-6}$ & $2.586 \times 10^{-6}$ & $2.586 \times 10^{-6}$ \\
\hline $\mathrm{ab} 4$ & $1.295 \times 10^{-5}$ & $1.295 \times 10^{-5}$ & $1.295 \times 10^{-5}$ & $6.467 \times 10^{-6}$ & $6.467 \times 10^{-6}$ & $6.467 \times 10^{-6}$ & $2.586 \times 10^{-6}$ & $2.586 \times 10^{-6}$ & $2.586 \times 10^{-6}$ \\
\hline
\end{tabular}

B.4. Fourth order - errors for $b_{n}(t)$.

\begin{tabular}{|c|c|c|c|c|c|c|c|c|c|}
\hline quad/Sol. & & $\mathrm{T}=1000$ & & & $\mathrm{~T}=2000$ & & & $\mathrm{~T}=5000$ & \\
\hline $\mathrm{dT}$ & 0.01 & 0.001 & 0.0001 & 0.01 & 0.001 & 0.0001 & 0.01 & 0.001 & 0.0001 \\
\hline rk4 & $2.847 \times 10^{-7}$ & $1.003 \times 10^{-9}$ & $1.022 \times 10^{-9}$ & $6.441 \times 10^{-7}$ & $1.373 \times 10^{-10}$ & $2.712 \times 10^{-9}$ & $2.228 \times 10^{-6}$ & $9.067 \times 10^{-10}$ & $1.857 \times 10^{-8}$ \\
\hline rk4qp & $3.25 \times 10^{-7}$ & $1.003 \times 10^{-9}$ & $1.002 \times 10^{-9}$ & $7.313 \times 10^{-7}$ & $5.296 \times 10^{-11}$ & $2.053 \times 10^{-11}$ & $2.511 \times 10^{-6}$ & $1.454 \times 10^{-10}$ & $6.31 \times 10^{-11}$ \\
\hline rkf 45 & $6.737 \times 10^{-8}$ & $1.002 \times 10^{-9}$ & $1.023 \times 10^{-9}$ & $1.667 \times 10^{-7}$ & $1.186 \times 10^{-10}$ & $2.718 \times 10^{-9}$ & $6.679 \times 10^{-7}$ & $8.814 \times 10^{-10}$ & $1.86 \times 10^{-8}$ \\
\hline $\mathrm{ab} 4$ & $3.512 \times 10^{-3}$ & $3.508 \times 10^{-5}$ & $3.505 \times 10^{-7}$ & $6.989 \times 10^{-3}$ & $6.989 \times 10^{-5}$ & $6.981 \times 10^{-7}$ & $1.747 \times 10^{-2}$ & $1.751 \times 10^{-4}$ & $1.749 \times 10^{-6}$ \\
\hline
\end{tabular}

\begin{tabular}{|c|c|c|c|c|c|c|c|c|c|}
\hline quad/Disp. & & $\mathrm{T}=1000$ & & & $\mathrm{~T}=2000$ & & & $\mathrm{~T}=5000$ & \\
\hline dT & 0.01 & 0.001 & 0.0001 & 0.01 & 0.001 & 0.0001 & 0.01 & 0.001 & 0.0001 \\
\hline rk4 & $1.348 \times 10^{-6}$ & $3.368 \times 10^{-9}$ & $3.399 \times 10^{-9}$ & $2.706 \times 10^{-6}$ & $7.313 \times 10^{-9}$ & $8.297 \times 10^{-9}$ & $6.593 \times 10^{-6}$ & $1.201 \times 10^{-8}$ & $7.663 \times 10^{-8}$ \\
\hline rk4qp & $1.358 \times 10^{-6}$ & $3.369 \times 10^{-9}$ & $3.406 \times 10^{-9}$ & $2.724 \times 10^{-6}$ & $7.302 \times 10^{-9}$ & $7.283 \times 10^{-9}$ & $6.632 \times 10^{-6}$ & $1.119 \times 10^{-8}$ & $1.117 \times 10^{-8}$ \\
\hline rkf45 & $2.101 \times 10^{-7}$ & $3.413 \times 10^{-9}$ & $3.399 \times 10^{-9}$ & $4.185 \times 10^{-7}$ & $7.276 \times 10^{-9}$ & $8.305 \times 10^{-9}$ & $1.022 \times 10^{-6}$ & $1.175 \times 10^{-8}$ & $7.646 \times 10^{-8}$ \\
\hline$a b 4$ & $4.187 \times 10^{-4}$ & $3.77 \times 10^{-6}$ & $3.745 \times 10^{-8}$ & $5.455 \times 10^{-4}$ & $4.51 \times 10^{-6}$ & $4.83 \times 10^{-8}$ & $7.542 \times 10^{-4}$ & $4.992 \times 10^{-6}$ & $1.231 \times 10^{-7}$ \\
\hline
\end{tabular}




\begin{tabular}{|c|c|c|c|c|c|c|c|c|c|}
\hline dirac/Sol. & & $\mathrm{T}=1000$ & & & $T=2000$ & & & $T=5000$ & \\
\hline $\mathrm{dT}$ & 0.01 & 0.001 & 0.0001 & 0.01 & 0.001 & 0.0001 & 0.01 & 0.001 & 0.0001 \\
\hline rk4 & $1.164 \times 10^{-3}$ & $1.46 \times 10^{-8}$ & $1.849 \times 10^{-11}$ & $1.071 \times 10^{-2}$ & $1.202 \times 10^{-7}$ & $1.683 \times 10^{-10}$ & $6.202 \times 10^{-2}$ & $6.725 \times 10^{-7}$ & $9.511 \times 10^{-10}$ \\
\hline rk4qp & $1.503 \times 10^{-3}$ & $1.915 \times 10^{-8}$ & $1.413 \times 10^{-11}$ & $1.382 \times 10^{-2}$ & $1.565 \times 10^{-7}$ & $1.287 \times 10^{-10}$ & $7.915 \times 10^{-2}$ & $8.697 \times 10^{-7}$ & $9.272 \times 10^{-11}$ \\
\hline rkf45 & $5.764 \times 10^{-4}$ & $6.248 \times 10^{-9}$ & $3.149 \times 10^{-11}$ & $5.305 \times 10^{-3}$ & $5.547 \times 10^{-8}$ & $8.918 \times 10^{-11}$ & $3.248 \times 10^{-2}$ & $3.262 \times 10^{-7}$ & $3.574 \times 10^{-10}$ \\
\hline $\mathrm{ab4}$ & $9.661 \times 10^{-2}$ & $3.876 \times 10^{-4}$ & $3.889 \times 10^{-6}$ & $9.443 \times 10^{-1}$ & $1.794 \times 10^{-3}$ & $1.809 \times 10^{-5}$ & 1.495 & $4.31 \times 10^{-3}$ & $4.382 \times 10^{-5}$ \\
\hline
\end{tabular}

\begin{tabular}{|c|c|c|c|c|c|c|c|c|c|}
\hline dirac/Disp. & & $\mathrm{T}=1000$ & & & $\mathrm{~T}=2000$ & & & $T=5000$ & \\
\hline $\mathrm{dT}$ & 0.01 & 0.001 & 0.0001 & 0.01 & 0.001 & 0.0001 & 0.01 & 0.001 & 0.0001 \\
\hline rk4 & $3.579 \times 10^{-6}$ & $1.693 \times 10^{-10}$ & $2.559 \times 10^{-10}$ & $7.044 \times 10^{-6}$ & $3.165 \times 10^{-10}$ & $8.138 \times 10^{-10}$ & $1.699 \times 10^{-5}$ & $1.126 \times 10^{-9}$ & $6.312 \times 10^{-9}$ \\
\hline rk4qp & $4.905 \times 10^{-6}$ & $1.876 \times 10^{-10}$ & $6.095 \times 10^{-11}$ & $9.656 \times 10^{-6}$ & $3.43 \times 10^{-10}$ & $8.417 \times 10^{-11}$ & $2.33 \times 10^{-5}$ & $8.094 \times 10^{-10}$ & $1.479 \times 10^{-10}$ \\
\hline rkf45 & $1.464 \times 10^{-6}$ & $3.167 \times 10^{-11}$ & $2.43 \times 10^{-10}$ & $2.938 \times 10^{-6}$ & $3.728 \times 10^{-11}$ & $9.681 \times 10^{-10}$ & $7.185 \times 10^{-6}$ & $3.512 \times 10^{-10}$ & $6.348 \times 10^{-9}$ \\
\hline $\mathrm{ab} 4$ & $5.444 \times 10^{-3}$ & $5.106 \times 10^{-5}$ & $5.111 \times 10^{-7}$ & $6.308 \times 10^{-3}$ & $5.581 \times 10^{-5}$ & $5.592 \times 10^{-7}$ & $7.896 \times 10^{-3}$ & $6.046 \times 10^{-5}$ & $6.104 \times 10^{-7}$ \\
\hline
\end{tabular}

\begin{tabular}{|c|c|c|c|c|c|c|c|c|c|}
\hline double/Sol. & & $T=1000$ & & & $T=2000$ & & & $T=5000$ & \\
\hline$\overline{d T}$ & 0.01 & 0.001 & 0.0001 & 0.01 & 0.001 & 0.0001 & 0.01 & 0.001 & 0.0001 \\
\hline rk4 & $1.001 \times 10^{-7}$ & $2.425 \times 10^{-11}$ & $1.776 \times 10^{-10}$ & $2.006 \times 10^{-7}$ & $6.516 \times 10^{-11}$ & $5.24 \times 10^{-10}$ & $5.287 \times 10^{-7}$ & $3.88 \times 10^{-10}$ & $1.59 \times 10^{-8}$ \\
\hline rk4qp & $1.128 \times 10^{-7}$ & $9.553 \times 10^{-12}$ & $2.335 \times 10^{-12}$ & $2.255 \times 10^{-7}$ & $1.866 \times 10^{-11}$ & $6.009 \times 10^{-12}$ & $5.919 \times 10^{-7}$ & $4.644 \times 10^{-11}$ & $7.897 \times 10^{-11}$ \\
\hline rkf45 & $1.867 \times 10^{-8}$ & $9.875 \times 10^{-12}$ & $1.779 \times 10^{-10}$ & $3.842 \times 10^{-8}$ & $3.104 \times 10^{-11}$ & $5.248 \times 10^{-10}$ & $1.083 \times 10^{-7}$ & $2.96 \times 10^{-10}$ & $1.589 \times 10^{-8}$ \\
\hline $\mathrm{ab4}$ & $4.27 \times 10^{-3}$ & $4.283 \times 10^{-5}$ & $4.283 \times 10^{-7}$ & $8.434 \times 10^{-3}$ & $8.461 \times 10^{-5}$ & $8.459 \times 10^{-7}$ & $2.091 \times 10^{-2}$ & $2.098 \times 10^{-4}$ & $2.098 \times 10^{-6}$ \\
\hline
\end{tabular}

\begin{tabular}{|c|c|c|c|c|c|c|c|c|c|}
\hline double/Disp. & & $\mathrm{T}=1000$ & & & $\mathrm{~T}=2000$ & & & $\mathrm{~T}=5000$ & \\
\hline $\mathrm{dT}$ & 0.01 & 0.001 & 0.0001 & 0.01 & 0.001 & 0.0001 & 0.01 & 0.001 & 0.0001 \\
\hline rk4 & $1.275 \times 10^{-6}$ & $1.357 \times 10^{-10}$ & $7.93 \times 10^{-11}$ & $2.61 \times 10^{-6}$ & $2.795 \times 10^{-10}$ & $2.291 \times 10^{-10}$ & $6.431 \times 10^{-6}$ & $9.859 \times 10^{-10}$ & $1.544 \times 10^{-8}$ \\
\hline rk4qp & $1.278 \times 10^{-6}$ & $1.287 \times 10^{-10}$ & $8.07 \times 10^{-12}$ & $2.617 \times 10^{-6}$ & $2.611 \times 10^{-10}$ & $1.358 \times 10^{-11}$ & $6.448 \times 10^{-6}$ & $6.493 \times 10^{-10}$ & $4.824 \times 10^{-11}$ \\
\hline rkf45 & $1.956 \times 10^{-7}$ & $1.569 \times 10^{-11}$ & $8.033 \times 10^{-11}$ & $4.003 \times 10^{-7}$ & $2.324 \times 10^{-11}$ & $2.334 \times 10^{-10}$ & $9.861 \times 10^{-7}$ & $2.212 \times 10^{-10}$ & $1.546 \times 10^{-8}$ \\
\hline$a b 4$ & $3.616 \times 10^{-4}$ & $3.123 \times 10^{-6}$ & $3.126 \times 10^{-8}$ & $4.477 \times 10^{-4}$ & $3.446 \times 10^{-6}$ & $3.459 \times 10^{-8}$ & $6.343 \times 10^{-4}$ & $3.762 \times 10^{-6}$ & $5.233 \times 10^{-8}$ \\
\hline
\end{tabular}

\begin{tabular}{|c|c|c|c|c|c|c|c|c|c|}
\hline NoS/Sol. & & $\mathrm{T}=1000$ & & & $\mathrm{~T}=2000$ & & & $T=5000$ & \\
\hline $\mathrm{dT}$ & 0.01 & 0.001 & 0.0001 & 0.01 & 0.001 & 0.0001 & 0.01 & 0.001 & 0.0001 \\
\hline rk4 & $2.315 \times 10^{-10}$ & $8.416 \times 10^{-11}$ & $8.496 \times 10^{-10}$ & $1.709 \times 10^{-10}$ & $1.501 \times 10^{-10}$ & $1.363 \times 10^{-9}$ & $1.287 \times 10^{-10}$ & $4.976 \times 10^{-10}$ & $5.047 \times 10^{-9}$ \\
\hline rk4qp & $2.47 \times 10^{-10}$ & $2.2 \times 10^{-11}$ & $2.201 \times 10^{-11}$ & $1.786 \times 10^{-10}$ & $1.828 \times 10^{-11}$ & $1.828 \times 10^{-11}$ & $1.21 \times 10^{-10}$ & $1.335 \times 10^{-11}$ & $1.335 \times 10^{-11}$ \\
\hline rkf45 & $4.745 \times 10^{-11}$ & $8.395 \times 10^{-11}$ & $8.496 \times 10^{-10}$ & $4.034 \times 10^{-11}$ & $1.497 \times 10^{-10}$ & $1.363 \times 10^{-9}$ & $6.015 \times 10^{-11}$ & $4.979 \times 10^{-10}$ & $5.047 \times 10^{-9}$ \\
\hline$a b 4$ & $1.246 \times 10^{-5}$ & $1.24 \times 10^{-7}$ & $1.511 \times 10^{-9}$ & $1.175 \times 10^{-5}$ & $1.168 \times 10^{-7}$ & $1.788 \times 10^{-9}$ & $1.017 \times 10^{-5}$ & $1.011 \times 10^{-7}$ & $5.151 \times 10^{-9}$ \\
\hline
\end{tabular}

\begin{tabular}{|c|c|c|c|c|c|c|c|c|c|}
\hline NoS/Disp. & & $\mathrm{T}=1000$ & & & $\mathrm{~T}=2000$ & & & $\mathrm{~T}=5000$ & \\
\hline $\mathrm{dT}$ & 0.01 & 0.001 & 0.0001 & 0.01 & 0.001 & 0.0001 & 0.01 & 0.001 & 0.0001 \\
\hline rk4 & $1.282 \times 10^{-6}$ & $1.325 \times 10^{-10}$ & $4.284 \times 10^{-11}$ & $2.566 \times 10^{-6}$ & $2.653 \times 10^{-10}$ & $4.116 \times 10^{-11}$ & $6.524 \times 10^{-6}$ & $6.385 \times 10^{-10}$ & $2.428 \times 10^{-10}$ \\
\hline rk4qp & $1.284 \times 10^{-6}$ & $1.278 \times 10^{-10}$ & $9.143 \times 10^{-12}$ & $2.57 \times 10^{-6}$ & $2.546 \times 10^{-10}$ & $1.756 \times 10^{-11}$ & $6.532 \times 10^{-6}$ & $6.535 \times 10^{-10}$ & $3.917 \times 10^{-11}$ \\
\hline rkf45 & $1.971 \times 10^{-7}$ & $1.87 \times 10^{-11}$ & $4.349 \times 10^{-11}$ & $3.946 \times 10^{-7}$ & $3.901 \times 10^{-11}$ & $4.469 \times 10^{-11}$ & $1.003 \times 10^{-6}$ & $1.359 \times 10^{-10}$ & $2.087 \times 10^{-10}$ \\
\hline ab4 & $9.781 \times 10^{-5}$ & $6.202 \times 10^{-7}$ & $6.199 \times 10^{-9}$ & $1.487 \times 10^{-4}$ & $6.416 \times 10^{-7}$ & $6.377 \times 10^{-9}$ & $3.154 \times 10^{-4}$ & $6.868 \times 10^{-7}$ & $6.585 \times 10^{-9}$ \\
\hline
\end{tabular}




\begin{tabular}{|c|c|c|c|c|c|c|c|c|c|}
\hline PureS/Sol. & & $\mathrm{T}=1000$ & & & $T=2000$ & & & $T=5000$ & \\
\hline $\mathrm{dT}$ & 0.01 & 0.001 & 0.0001 & 0.01 & 0.001 & 0.0001 & 0.01 & 0.001 & 0.0001 \\
\hline rk4 & $4.752 \times 10^{-9}$ & $1.161 \times 10^{-10}$ & $2.201 \times 10^{-10}$ & $9.436 \times 10^{-9}$ & $2.965 \times 10^{-10}$ & $2.74 \times 10^{-10}$ & $2.63 \times 10^{-8}$ & $2.681 \times 10^{-10}$ & $1.292 \times 10^{-9}$ \\
\hline rk4qp & $4.879 \times 10^{-9}$ & $1.034 \times 10^{-11}$ & $1.054 \times 10^{-11}$ & $9.72 \times 10^{-9}$ & $5.901 \times 10^{-12}$ & $7.241 \times 10^{-12}$ & $2.713 \times 10^{-8}$ & $2.156 \times 10^{-12}$ & $1.005 \times 10^{-11}$ \\
\hline rkf 45 & $7.937 \times 10^{-10}$ & $1.935 \times 10^{-11}$ & $8.682 \times 10^{-10}$ & $1.692 \times 10^{-9}$ & $2.888 \times 10^{-11}$ & $2.609 \times 10^{-9}$ & $5.421 \times 10^{-9}$ & $1.172 \times 10^{-10}$ & $1.264 \times 10^{-9}$ \\
\hline$a b 4$ & $7.724 \times 10^{-5}$ & $7.741 \times 10^{-7}$ & $7.675 \times 10^{-9}$ & $1.579 \times 10^{-4}$ & $1.583 \times 10^{-6}$ & $1.592 \times 10^{-8}$ & $4.002 \times 10^{-4}$ & $4.015 \times 10^{-6}$ & $3.878 \times 10^{-8}$ \\
\hline PureS/Disf & & $\mathrm{T}=1000$ & & & $\mathrm{~T}=2000$ & & & $\mathrm{~T}=5000$ & \\
\hline $\mathrm{dT}$ & 0.01 & 0.001 & 0.0001 & 0.01 & 0.001 & 0.0001 & 0.01 & 0.001 & 0.0001 \\
\hline rk4 & $2.589 \times 10^{-}$ & $2.589 \times 10^{-5}$ & $2.589 \times 10^{-5}$ & $1.293 \times 10^{-5}$ & $1.293 \times 10^{-5}$ & $1.293 \times 10^{-5}$ & $5.172 \times 10^{-6}$ & $5.172 \times 10^{-6}$ & $5.172 \times 10^{-6}$ \\
\hline rk4qp & $2.589 \times 10^{-}$ & $2.589 \times 10^{-5}$ & $2.589 \times 10^{-5}$ & $1.293 \times 10^{-5}$ & $1.293 \times 10^{-5}$ & $1.293 \times 10^{-5}$ & $5.172 \times 10^{-6}$ & $5.172 \times 10^{-6}$ & $5.172 \times 10^{-6}$ \\
\hline rkf45 & $2.589 \times 10^{-}$ & $2.589 \times 10^{-5}$ & $2.589 \times 10^{-5}$ & $1.293 \times 10^{-5}$ & $1.293 \times 10^{-5}$ & $1.293 \times 10^{-5}$ & $5.172 \times 10^{-6}$ & $5.172 \times 10^{-6}$ & $5.172 \times 10^{-6}$ \\
\hline$a b 4$ & $2.589 \times 10^{-}$ & $2.589 \times 10^{-5}$ & $2.589 \times 10^{-5}$ & $1.294 \times 10^{-5}$ & $1.293 \times 10^{-5}$ & $1.293 \times 10^{-5}$ & $5.173 \times 10^{-6}$ & $5.172 \times 10^{-6}$ & $5.172 \times 10^{-6}$ \\
\hline
\end{tabular}




\section{REFERENCES}

[1] TodaBenchmarking. https://bitbucket.org/trogdon/todabenchmarking. Accessed: 2017-09-12. 12

[2] D. Bilman And S. Konstantinou-Rizos, Discrete Integrable Systems, Darboux Transformations, and Yang-Baxter Maps, in Symmetries Integr. Differ. Equations, D. Levi, R. Rebelo, and P. Winternitz, eds., Springer International Publishing, Cham, 2017, pp. 195-260. 3

[3] D. Bilman AND I. NenciU, On the evolution of scattering data under perturbations of the Toda lattice, Phys. D Nonlinear Phenom., 330 (2016), pp. 1-16. 2, 12

[4] D. BILMAN AND T. TROGDON, ISTPackage. https://bitbucket.org/trogdon/istpackage, 2013.3

[5] D. Bilman And T. Trogdon, Numerical Inverse Scattering for the Toda Lattice, Commun. Math. Phys., 352 (2017), pp. 805-879. 2, 3, 8, 12

[6] J. Butcher, A history of Runge-Kutta methods, Appl. Numer. Math., 20 (1996), pp. 247-260. 5

[7] _ Numerical methods for ordinary differential equations in the 20th century, J. Comput. Appl. Math., 125 (2000), pp. 1-29. 2

[8] E. FeHLBERG, Klassische Runge-Kutta-Formeln vierter und niedrigerer Ordnung mit Schrittweiten-Kontrolle und ihre Anwendung auf Wärmeleitungsprobleme, Computing, 6 (1970), pp. 61-71. 5, 12

[9] I. Fermi, P. PASta, S. Ulam, and M. Tsingou, STUDIES OF THE NONLINEAR PROBLEMS, tech. rep., Los Alamos National Laboratory (LANL), Los Alamos, NM (United States), may 1955. 2

[10] H. FlaschKA, The Toda lattice. II. Existence of integrals, Phys. Rev. B, 9 (1974), pp. 1924-1925. 3

[11] J. GAison, S. Moskow, J. D. Wright, AND Q. ZHANG, Approximation of Polyatomic FPU Lattices by KdV Equations, Multiscale Model. Simul., 12 (2014), pp. 953-995. 2

[12] E. Hairer, S. Nørsett, And G. WAnner, Solving Ordinary Differential Equation I: Nonstiff Problems, SpringerVerlag, Berlin, 2 ed., 1993. 5

[13] A. HofFMAN AND J. D. WRIGHT, Nanopteron solutions of diatomic Fermi-Pasta-Ulam-Tsingou lattices with small massratio, Phys. D Nonlinear Phenom., (2017). 2

[14] T. E. Hull, W. H. EnRight, B. M. Fellen, And A. E. Sedgwick, Comparing Numerical Methods for Ordinary Differential Equations, SIAM J. Numer. Anal., 9 (1972), pp. 603-637. 2

[15] S. KAMVISSIS, On the long time behavior of the doubly infinite Toda lattice under initial data decaying at infinity, Comm. Math. Phys., 153 (1993), pp. 479-519. 8

[16] H. KRÜGER AND G. TESCHL, Long-time asymptotics of the Toda lattice for decaying initial data revisited, Rev. Math. Phys., 21 (2009), pp. 61-109. 8

[17] W. KUTTA, Beitrag zur näherungsweisen Integration totaler Differentialgleichungen, Z. Math. Phys., 46 (1901), pp. 435453. 5

[18] P. D. LAX, Integrals of nonlinear equations of evolution and solitary waves, Commun. Pure Appl. Math., 21 (1968), pp. $467-490.3$

[19] R. J. LeVeque, Finite Difference Methods for Ordinary and Partial Differential Equations, SIAM, Philadelphia, PA, 2007. 5

[20] S. ManaKov, Complete integrability and stochastization of discrete dynamical systems., Sov. Phys. JETP, 40 (1975), pp. 269-274. 3

[21] S. OLVER, RHPackage. http://www.maths.usyd.edu.au/u/olver/projects/RHPackage.html, 2010. 3

[22] _ - A general framework for solving Riemann-Hilbert problems numerically, Numer. Math., 122 (2012), pp. 305-340. 3

[23] S. Olver And T. Trogdon, Nonlinear Steepest Descent and Numerical Solution of Riemann-Hilbert Problems, Commun. Pure Appl. Math., 67 (2014), pp. 1353-1389. 2, 3

[24] C. RungE, Über die numerische Auflösung von Differentialgleichungen, Math. Ann., 46 (1895), pp. 167-178. 5

[25] M. Sato, B. E. Hubbard, A. J. Sievers, B. Ilic, D. A. Czaplewski, and H. G. Craighead, Observation of Locked Intrinsic Localized Vibrational Modes in a Micromechanical Oscillator Array, Phys. Rev. Lett., 90 (2003), p. 044102. 2

[26] M. TodA, Wave Propagation in Anharmonic Lattices, J. Phys. Soc. Japan, 23 (1967), pp. 501-506. 2

[27] T. TROGDON AND B. DECONINCK, A Riemann-Hilbert problem for the finite-genus solutions of the KdV equation and its numerical solution, Phys. D, 251 (2013), pp. 1-18. 3

[28] T. TROGDON AND S. Olver, Numerical inverse scattering for the focusing and defocusing nonlinear Schrödinger equations, Proc. R. Soc. A Math. Phys. Eng. Sci., 469 (2012), pp. 20120330-20120330. 3

[29] T. Trogdon And S. Olver, Riemann-Hilbert Problems, Their Numerical Solution and the Computation of Nonlinear Special Functions, SIAM, Philadelphia, PA, 2016. 3

[30] T. Trogdon, S. Olver, AND B. DeCONINCK, Numerical inverse scattering for the Korteweg-de Vries and modified Korteweg-de Vries equations, Phys. D, 241 (2012), pp. 1003-1025. 3

[31] A. Vainchtein, Y. Starosvetsky, J. D. Wright, And R. Perline, Solitary waves in diatomic chains, Phys. Rev. E, 93 (2016), p. 042210.2 
[32] N. J. ZABUSKY AND M. D. KRUSKAL, Interaction of "Solitons" in a Collisionless Plasma and the Recurrence of Initial States, Phys. Rev. Lett., 15 (1965), pp. 240-243. 2

Deniz Bilman, Department of Mathematics, University of Michigan, 530 Church Street, Ann Arbor, MI 48109

E-mail address: bilman@umich.edu

Thomas Trogdon, University of CALIFornia, IRvine, Rowland HALl, IRvine, CA 92697

E-mail address: ttrogdon@uci.edu 